\title{
Dynamic Dilution Exponent in Monodisperse Entangled Polymer Solutions
}

\author{
T. Shahid ${ }^{1,2}$, Q. Huang ${ }^{3}$, F. Oosterlinck ${ }^{4}$, C. Clasen ${ }^{1}$, E. van Ruymbeke ${ }^{2 *}$ \\ I: Department of Chemical Engineering, KU Leuven, 3001 Heverlee, Belgium \\ 2: Bio and Soft Matter, Institute of Condensed Matter and Nano-science (IMCN), Universite \\ catholique de Louvain, 1348 Louvain-La-Neuve, Belgium \\ 3. Department of Chemical and Biochemical Engineering, The Danish Polymer Centre, 2800 Kgs. \\ Lyngby, Denmark. \\ ${ }^{4}$ : Material Science Center, DSM Research Geleen, The Netherlands
}

\begin{abstract}
We study and model the linear viscoelastic properties of several entangled semi-dilute and concentrated solutions of linear chains of different molar masses and at different concentrations dissolved in their oligomers. We discuss the dilution effect by the oligomers on the entangled long chains. In particular, we investigate the influence of both concentration and molar mass on the value of the effective dynamic dilution exponent determined from the level of the storage plateau at low and intermediate frequencies. We show that the experimental results can be quantitatively explained by considering the tension reequilibration process along the chains, in agreement with [van Ruymbeke et al., Macromol. 2014], i.e. by considering that the real dilution exponent $\alpha$ is always equal to 1 , while larger values of dilution exponent $(1<\alpha<1.3)$ found experimentally are attributed to the enhanced relaxation of the long chain extremities. Then we discuss the influence of the polymer concentration on the terminal relaxation time of the solutions and how this can be modelled by the enhanced contour length fluctuations process (CR-CLF). We point out that this larger dilution effect is not only a function of concentration but also depends on the molar mass of the chains.

While the proposed approach successfully explains the viscoelastic properties of a large number of semi-dilute solutions of polymers in their own oligomers, important discrepancies are found for semi-dilute entangled polymers in small-molecule theta or good solvents. Possible explanations for the differences between these sample sets are proposed, based on a comparison of their viscoelastic behavior.
\end{abstract}




\section{Introduction}

Today, the linear viscoelastic properties of linear entangled polymer melts are quite well understood and can be predicted on a quantitative level, based on coarse-grained models such as tube-models ${ }^{1-4}$ or slip-link models ${ }^{5-8}$. Beside the melt state, these models can also be used to describe the properties of entangled polymer solutions or of long polymer chains diluted in a matrix of very short chains or oligomers ${ }^{9-11}$. However, this requires accounting for constraint release mechanisms in order to describe the influence of the fast motion of shorter and oligomeric chains on the relaxation of the long chains. To this end, most of the present tube models are using the concept of dynamic tube dilution (DTD) proposed by Marrucci et al. ${ }^{12}$, according to which the effective diameter of the tube, $a$, which represents the constraining effect of the surrounding chains on the motion of a specific molecule, is increasing if the polymer concentration is reduced, as well as if part of the sample is moving fast enough so that they do not constrain the motions of the long chains anymore ${ }^{1,2}$. Consequently, the entanglements involving these chains can be ignored and the effective average molar mass between two entanglements, $M_{e}$, becomes larger ${ }^{11,12}$ :

$a(t)=\frac{a_{0, \text { melt }}}{(c . \phi(t))^{\frac{\alpha}{2}},}$,
$M_{e}(t)=\frac{M_{e 0, \text { melt }}}{(c . \phi(t))^{\alpha}}$,

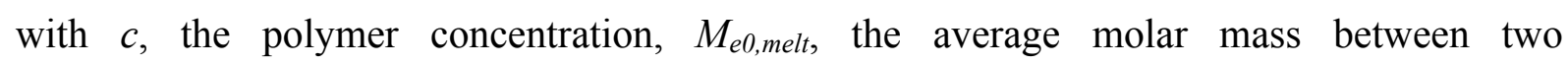
entanglements in the melt state at time $t=0, \phi(t)$, the fraction of unrelaxed polymer which still contribute to the constraining tube at time $t$, and $\alpha$, the dynamic dilution exponent. As a consequence of rescaling $M_{e}$, the plateau modulus $G_{N}{ }^{0}$ and the relaxation modulus $G_{\mathrm{d}}(t)$ of an entangled polymer solution can be defined as ${ }^{13}$ :

$G_{N}^{0}=\frac{\rho R T}{M_{e}}=\frac{c \rho_{0} R T}{\frac{M_{e 0, m e l t}}{c^{\alpha}}}=G_{N, m e l t}^{0} c^{\alpha+1}$

$G_{d}(t)=\left(G_{N}^{0} \cdot \phi(t)^{\alpha}\right) \cdot \phi(t)=G_{N, m e l t}^{0} c^{\alpha+1} \phi(t)^{\alpha+1}$ 
with $\rho$, the density of the solution, and $\rho_{0}$ and $G_{N, \text { melt }}^{0}$, the density and plateau modulus of the corresponding melt.

Constraint Release (CR) mechanisms also affect the terminal relaxation time, $\tau_{\text {rel }}(M)$, of the polymer solutions and in particular their reptation time, $\tau_{\text {rept }}(M) .{ }^{12}$ The latter can be compared to the initial reptation time in the melt, $\tau_{\text {rept } 0 \text {,melt }}(M)$, by accounting for the fact that the corresponding Rouse time of an entanglement segment, $\tau_{e}$, is becoming longer (since $M_{e}$ is longer), while the number of entanglement segments along the chain, $Z$, is decreasing (since $\left.Z=M / M_{e}\right)$ :

$\tau_{\text {rept }}(M)=3 \tau_{e} Z^{3}=3\left(\frac{\tau_{e 0, \text { melt }}}{(c . \phi(t))^{2 \alpha}}\right)\left(Z_{0, \text { melt }}(c . \phi(t))^{\alpha}\right)^{3}=\tau_{\text {rept } 0, \text { melt }}(M)(c . \phi(t))^{\alpha}$

The $c^{\alpha}$ scaling of the reptation time can also be found by considering that reptation time is proportional to $L^{2} / D$, with $L$, the length of the primitive path of the chain (which scales with $\left.c^{\alpha / 2}\right)$, and $D$, the diffusion coefficient.

It must be noted that, in addition to the reptation process, a contour length fluctuations process (CLF) must be taken into account in order to determine the terminal relaxation time of the chain. This further speeds up the relaxation of the chains (see Section III).

The objective of this work is to discuss the value of the dynamic dilution exponent $\alpha$ in case of long linear chains diluted in oligomers or in real solvents. While this parameter should be $a$ priori equal to 1 if we consider that entanglements are binary contacts, several works have shown that, in the specific case of entangled solutions, its effectively observed value is rather equal to 4/3. ${ }^{14-21}$ This effective value was determined based on experimental data, by looking at the scaling of the level of the plateau modulus in the terminal regime with the concentration $c$, based on Eq. 3, where the plateau modulus can be conveniently approximated as being 3.56 times the value of the loss moduli at its maximum $G^{\prime \prime}{ }_{\max }{ }^{18}$ (or 3.24 in ref.[14]) in case of monodisperse samples. In the following we will refer to an effective $\alpha$ whenever this is obtained from the scaling of $\mathrm{G}{ }^{\prime}{ }_{\max }$ (or $G_{\delta}$ see below) with concentration.

An explanation of the observed effective value of $\alpha$ close to 1.3 (or 4/3) was suggested by Colby and Rubinstein ${ }^{17}$, based on the level of the second (low frequency) plateau modulus of 
the longer chains and its scaling with concentration. They proposed that an entanglement is created only if a fixed number of binary contacts between the chains is reached. Based on this assumption, they estimated the size of the 'blob' needed in order to ensure the creation of an effective entanglement at equilibrium, and they could show that such a blob size is proportional to $c^{-2 / 3}$. As for the entangled solution, an effective value of $\alpha$ of $4 / 3$ was also found for polymer blends composed of two monodisperse linear polymers ${ }^{15}$ with very different molar masses. However, in other cases such as for binary blends of linear chains with similar molar masses, or for branched molecules, the effective dynamic dilution exponent was found to be equal to 1. ${ }^{9,22-25}$ Then again, from results obtained with slip-link simulations it appears that the (second) plateau modulus scales with concentration as if the dynamic dilution exponent was equal to an effective value of $4 / 3$, despite the fact that its actual value was fixed to 1 in the model itself ${ }^{7,8}$. Thus, today, there is not a real consensus on what the effective value of $\alpha$ should be and how it is related to the actual value.

Recently, it was proposed by van Ruymbeke and co-workers ${ }^{11}$ that the actual dilution exponent $\alpha$ should be equal to 1, whatever the composition of the sample is. In order to explain a possible lowering of the plateau modulus with concentration (that would lead, following Eq. (3), to an increase of the effective $\alpha$ to values above 1), for solutions of longer polymer chains in their shorter oligomers the existence of an extra relaxation process was pointed out, originating from a tension re-equilibration along the chain ${ }^{26}$, which takes place at the time scale of the release/re-formation of the entanglements between a long and a short chain (i.e. a 'short-long' entanglement), and which leads to the loss of some initial 'long-long' entanglements at the chain extremities, independently from the reptation/fluctuations of the long chains. This process is usually slow and does not significantly affect the relaxation of the long component, leading to a clearly observable plateau modulus of this component and consequently to an experimentally determined effective $\alpha$ exponent equal to 1 . However, in specific cases, for example when the relaxation times of the short component is much shorter than the one of the long component, this process can speed up the relaxation of the long chain and consequently, the observable second plateau modulus is apparently lowered, leading to a scaling of $\mathrm{G}_{\mathrm{N}}{ }^{0}$ with concentration as if the effective dilution exponent $\alpha$ was equal to $4 / 3$.

In order to include this mechanism in tube models, a dual approach was proposed, which considers two different pictures related to two different length scales (tube diameters) and 
time scales ${ }^{11}$ : According to the first picture the long chains are relaxing in a thin tube of

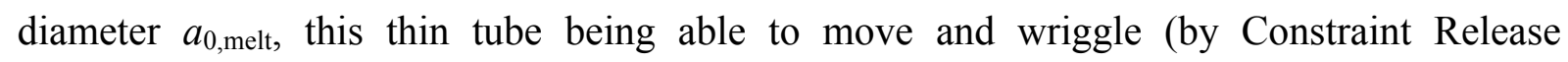
mechanisms) in a larger tube of diameter $a$ defined as ${ }^{7,12,27,28}$ :

$a=\frac{a_{0, m e l t}}{\left(v_{2}\right)^{\frac{\alpha}{2}}}$,

with $v_{2}$, the volume fraction of the long chains. In such a case, the reptation and fluctuation processes take place in a thin tube composed of entanglements from both long and short chains, and the corresponding main time unit is $\tau_{e 0 \text {, melt }}$. On the other hand, according to the second picture, the thin tube is not effective anymore and the long chains are moving and relaxing in a dilated tube of diameter $a .^{29}$ In such a case, the short chains can be compared to a solvent except for the fact that an extra friction, coming from their slower motions, must be taken into account to determine how fast the long chains can move. Indeed, the long chains can only move at the time scale of the release/re-formation (or 'blinking') of the short-long entanglements, which slows down their relaxation processes (compared to a real solvent), and the importance of the delay depends on the relaxation time of the short chains ${ }^{11}$. This requires defining a new Rouse relaxation time $\tau_{\text {long-long eql }}$, which represents the necessary time for a long-long entanglement of mass $M_{e}\left(=M_{e 0, \text { melt }} / v_{2}{ }^{\alpha}\right)$ to relax by a Rouse process, and which takes into account this possible delay from the motion of the short chains. If the long chains contain at least two long-long entanglement segments, this time has been defined as ${ }^{11}$ :

$\tau_{\text {long-long eql }}=\max \left(\frac{\tau_{e 0, \text { melt }}}{v_{2}^{2 \alpha}}, \tau_{\text {short-long }}\left(\frac{v_{1}}{v_{2}}\right)^{2 \alpha}\right)$

with $v_{1}$, the volume fraction of the short chains and $\tau_{\text {short-long, }}$ the average relaxation time of a short-long entanglement. Thus, according to this second picture, the chains are moving in a dilated tube, which speeds up their reptation and fluctuations processes, but their internal clock', defined by $\tau_{\text {long-long eql, }}$, can become slower than the intrinsic Rouse time of this segment, since it depends on the motion of the short chains. Therefore, depending on which process is faster and dominates, the long chains relaxation will be better described according to the first or the second tube picture. 
In ref. [11], it was shown that for binary blends of linear chains, where the second picture (i.e. long chains moving in a dilated tube with an internal clock defined by Eq. 7) is applicable, the long chains are relaxing faster than expected from the classical tube picture, and the observable part of the second plateau modulus is effectively lowered, resulting in an effective dynamic dilution exponent $\alpha$ larger than 1 when this is obtained from the scaling the second plateau modulus with concentration following Eq. 3. Furthermore, it was shown that in the extreme case of binary blends composed of very short unentangled chains (or oligomers), the 'second picture' must be considered, but with the specificity that there is no extra delay in the Rouse time of a long-long entanglement segment. Indeed, in such a case, the limiting relaxation process of a long-long entanglement is its intrinsic Rouse relaxation. Therefore, eq. 7 reduces to:

$\tau_{\text {long-long eql }}=\frac{\tau_{e 0, \text { melt }}}{v_{2}{ }^{2 \alpha}}$,

and an effective $\alpha$ exponent of $4 / 3$ was found for a specific set of samples, even if $\alpha$ was fixed to 1 in equation (1), (6) and (8). ${ }^{11}$

Thus, by considering that the long chains in binary blends can relax according to two different length-(and time-) scales, which corresponds to two different tube diameters, the experimental data of many binary blends could be correctly described, based on an actual $\alpha$ value of 1 .

However, several questions remain that we would like to address in the present work. In particular, we would like to test this approach on a larger set of semi-dilute or concentrated solutions of entangled polymers, diluted either in oligomers or in real solvents, in order to validate our picture. In particular, while it is expected that for such samples the second picture, which considers the long chains moving in a dilated tube, should always be active, it is to our opinion not obvious that the effective $\alpha$ for entangled solutions should always be equal of $4 / 3$ (as proposed in literature for entangled solutions ${ }^{14-18}$ ). The effective $\alpha$ obtained from the scaling of $G{ }^{\prime}{ }_{\max }$ with concentration should rather depend on the equilibration state of the long chain ${ }^{11}$ in the terminal regime where the effective plateau modulus (which is approximated by $3.56 G^{\prime \prime}{ }_{\max }$ ) is determined. Also, we would like to investigate if an effective $\alpha$ larger than 1 is also obtained when using the value of the storage modulus detected at the higher frequency $\omega_{\delta}$, where the $\tan \delta$ curve exhibits a minimum, a criterion that is often used 
to define the plateau modulus. As a last point, we would like to investigate the role of the solvent in the terminal relaxation time of the samples.

In order to address these points, a new set of model polymer solutions based on a very long polystyrene linear chains diluted in oligomers at several concentrations has been prepared and measured, and the corresponding linear viscoelastic data are discussed, also in comparison to other sets of samples presented in literature ${ }^{10,14,19,30-32}$. To do so, we use our tube-based model $^{11}$ in order to determine, based on both experimental and theoretical data, how the value of the effective $\alpha$ is affected by the chains concentration, their molar mass and nature of the solvent.

The paper is organized as follow. At first, in Section II the material used for this study as well as the experimental techniques are presented. Then, in Section III we present the model used for binary blends of linear chains, and reduced to the specific case of entangled polymer solutions. The results are presented and discussed in Section IV, where the experimental and theoretical data for several sets of samples are compared. The comparisons are first made for entangled polymers diluted in oligomers, and subsequently for the case of entangled polymers diluted in small-molecule solvents. Finally conclusions are drawn in Section V.

\section{Materials and experimental details}

\section{II.1. Materials and sample preparation}

The samples investigated in this work are monodisperse linear polystyrene chains either diluted in styrene oligomers or in the melt state. Some of these samples have been studied in previous works ${ }^{30-32}$ and their viscoelastic data have already been presented in literature. These samples are listed in Table 1, together with their corresponding references. Among them, there are several monodisperse PS samples in the melt state, which will be mainly used to define and validate the material parameters used in our tube model. There is also a set of PS samples diluted in oligomers, which has been presented in ref. [30] by Huang et al., and which has been designed in order to ensure that each of these samples contains a similar number of entanglements. 
Besides these two first sets of samples, a new set of entangled solutions has been designed and prepared for the purpose of this study. These new samples consist on a very high molar mass PS polymer $\left(\mathrm{M}_{\mathrm{w}}=820 \mathrm{~kg} / \mathrm{mol}\right)$ diluted in oligomers $\left(\mathrm{M}_{\mathrm{w}}=8.8 \mathrm{~kg} / \mathrm{mol}\right)$ at different concentrations, ranging from $3 \mathrm{wt} \%$ to $100 \mathrm{wt} \%$ of polymer (see Table 1).

Table 1: Main characteristics of the samples used in this work

\begin{tabular}{|c|c|c|c|c|c|}
\hline & $\mathrm{M}_{\mathrm{w}}$ & $\mathrm{M}_{\mathrm{n}}$ & PDI & Concentration & Ref. \\
\hline OS4 & 3,630 & 3424 & 1.06 & - & 30 \\
\hline OS8.8 & 8,800 & 8000 & 1.1 & - & - \\
\hline PS50 & 51,700 & 50,380 & 1.026 & $100 \%$ & 33 \\
\hline PS100 & 102,800 & 100,600 & 1.022 & $100 \%$ & 33 \\
\hline PS200 & 200,000 & 192,000 & 1.04 & $100 \%$ & 33 \\
\hline PS285 & 285,000 & 261,500 & 1.09 & $100 \%$ & 31 \\
\hline PS390 & 390,000 & 367,000 & 1.06 & $100 \%$ & 33 \\
\hline PS545 & 545,000 & 486,600 & 1.12 & $100 \%$ & 31 \\
\hline PS545 & 545,000 & 486,600 & 1.12 & $52 \%$ in OS4 & 30 \\
\hline PS900 & 910,000 & 784,500 & 1.16 & $33 \%$ in OS4 & 30 \\
\hline PS1760 & $1,880,000$ & $1,663,700$ & 1.13 & $18 \%$ in OS4 & 30 \\
\hline PS3200 & $3.140,000$ & $2.275,000$ & 1.38 & $13 \%$ in OS4 & 30 \\
\hline PS3900 & $3.900,000$ & $3,714,300$ & 1.05 & $10 \%$ in DEP & 34 \\
\hline PS820 & 820,000 & 805,000 & 1.02 & $\begin{array}{c}100 \%, 80 \%, 50 \%, 40 \%, 24 \% \\
10 \%, 5 \%, 3 \% \text { in OS } 8.8\end{array}$ & - \\
\hline PS8420 & 8420,000 & 7200,000 & 1.17 & $\begin{array}{l}5 \%, 4 \%, 3 \%, 2.5 \% \text { in } \\
\text { chlorinated biphenyl }\end{array}$ & 19 \\
\hline PBD410 & 411,500 & 410,800 & 1.01 & $100 \%, 5 \%$ in PBD $3 \mathrm{~kg} / \mathrm{mol}$ & 10 \\
\hline PBD925 & 925,000 & / & $<1.1$ & $\begin{array}{c}52.3 \%, 30.6 \%, 15.7 \%, 8.06 \% \\
3.08 \%, 2.14 \% \text { in dioctyl } \\
\text { phthalate (DOP) }\end{array}$ & 14 \\
\hline
\end{tabular}

Both the polystyrene and oligomers were purchased from Polymer Source (Montreal, Canada). The molar mass of the oligomers has been fixed in order to be short enough to be considered as a solvent, while long enough in order to avoid a too large decrease of their glass transition temperature compared to the high molecular weight sample (see Section II.2.). The 
high molar mass sample was selected to ensure a polydispersity as low as possible. Its molar mass and polydispersity were checked by Field Flow Fractionation (FFF) coupled with Multiangle light Scattering (MALS) (performed at BASF, Germany). The results were found to be consistent with the ones received from Polymer Source, and are presented in Table 1.

The solutions were prepared by diluting the high molecular weight Polystyrene, PS820, in the oligomeric styrene, OS8.8, at seven different weight fractions ranging from very concentrated to semi-dilute and dilute solutions regimes.

A 4-step procedure inspired by ref [30] was used to prepare the solutions. For this we dissolved first the polystyrene and the oligomer according to the desired weight fractions together in THF. The mixture was stirred at room temperature overnight to ensure that the two components were well dissolved and mixed. In a next step, the THF solution was added to the non-solvent methanol drop by drop, using a burette. The precipitated polymer/oligomer blend was then separated and collected using paper filtration. Finally, the blends were dried at $70^{\circ} \mathrm{C}$ under vacuum for 4 days to remove remaining traces of the solvents.

In addition to polymer/oligomers blends, semi-dilute or concentrated solutions of polymers diluted in small molecule solvents, which are investigated in Section IV.4, are also listed in Table 1 with their corresponding references.

\section{II.2. Linear viscoelastic properties}

The linear viscoelastic properties of the polystyrene melt and the solutions were measured by small amplitude oscillatory shear (SAOS) tests. These tests were performed on an AR2000 rheometer using an $8 \mathrm{~mm}$ parallel plate geometry and under constant nitrogen supply. Each SAOS test contained three different sub-tests. First, an amplitude sweep test was done to estimate the linear region of each sample. This was done at $1 \mathrm{rad} / \mathrm{s}$ and up to $10 \%$ of initial amplitude of the sample. Then a time sweep test was done to make sure the sample was well equilibrated with the experimental temperature and all the residual stresses were relaxed. Finally, a frequency sweep test was done to determine the linear viscoelastic data of each sample. 
For the PS melt, the frequency sweep test was done at four different temperatures ranging from $140^{\circ} \mathrm{C}$ to $190^{\circ} \mathrm{C}$, while the solutions were measured at three different temperatures ranging from $130^{\circ} \mathrm{C}$ to $170^{\circ} \mathrm{C}$. Subsequently the data for each temperature was shifted per sample onto a single master curve using the time-temperature superposition (TTS) procedure $^{35}$.

\section{Working under the iso- $T_{g}$ condition:}

As shown in Table 2, the glass transition temperature $T_{g}$ of the polymer solutions, which has been determined by Differential Scanning Calorimetry (DSC), varies with the polymer concentration. This difference must be taken into account in order to allow a comparison of the viscoelastic responses of the different solutions. To this end, as proposed in ref. [36], master-curves have been created at different reference temperatures $T_{\text {ref., }}$ such that these temperatures correspond to iso- $T_{g}$ condition, i.e. these temperatures are at a constant distance from the corresponding $T_{g}$ (i.e. $T_{\text {ref. }}-T_{g}=$ constant $)$.

Table 2: Tg of the different solutions.

\begin{tabular}{|l|l|l|}
\hline Solutions & Number of entanglements $^{\mathbf{a}}$ & $\left.\mathbf{T}_{\mathbf{g}} \mathbf{(}^{\mathbf{0}} \mathbf{C}\right)$ \\
\hline OS8.8 melt state & - & 94.6 \\
\hline OS8.8 + PS820 (3\%) & 2 & 98.1 \\
\hline OS8.8 + PS820 (5\%) & 3 & 98.4 \\
\hline OS8.8 + PS820 (10\%) & 5 & 98.6 \\
\hline OS8.8 + PS820 (25\%) & 14 & 100.4 \\
\hline OS8.8 + PS820 (40\%) & 22 & 101.3 \\
\hline OS8.8 + PS820 (50\%) & 27 & 102.3 \\
\hline OS8.8 + PS820 (80\%) & 44 & 104.6 \\
\hline PS820 melt state & 55 & 106.6 \\
\hline
\end{tabular}

${ }^{a}:$ The number of entanglements are determined based on $M_{e}=15 \mathrm{~kg} / \mathrm{mol}$.

In this paper the experimental data of the PS820 in the melt state at $130^{\circ} \mathrm{C}$ were chosen as the reference point, leading to a difference between the reference and glass transition temperature, $T_{\text {ref. }}-T_{g}$, of $23.4^{0} \mathrm{C}$. The storage and loss moduli of the other solutions measured at $130^{\circ} \mathrm{C}$ were then horizontally shifted in order to ensure a good overlap of the viscoelastic response in the high frequency region, in which the chain relaxation is controlled by local Rouse 
dynamics and will thus depend only on $\left(T_{\text {ref. }}-T_{g}\right)$. As discussed below, the validity of the shift factors to this specific reference temperature can then be confirmed by comparing their value to the shift factors vs temperature curve used to build the different TTS master-curves for the differently concentrated solutions.

\section{Master-curves:}

The shifted iso- $\mathrm{T}_{\mathrm{g}}$ data measured at $130^{\circ} \mathrm{C}$ are then used as reference curves to shift the other experimental data measured at higher temperatures and thus, to create general master-curves. It must be noted that no vertical shift has been considered here, since the range of temperature used is rather limited. In Figure 1, all shift factors used to create the master curves are plotted against $\left(T-T_{g}\right)$.

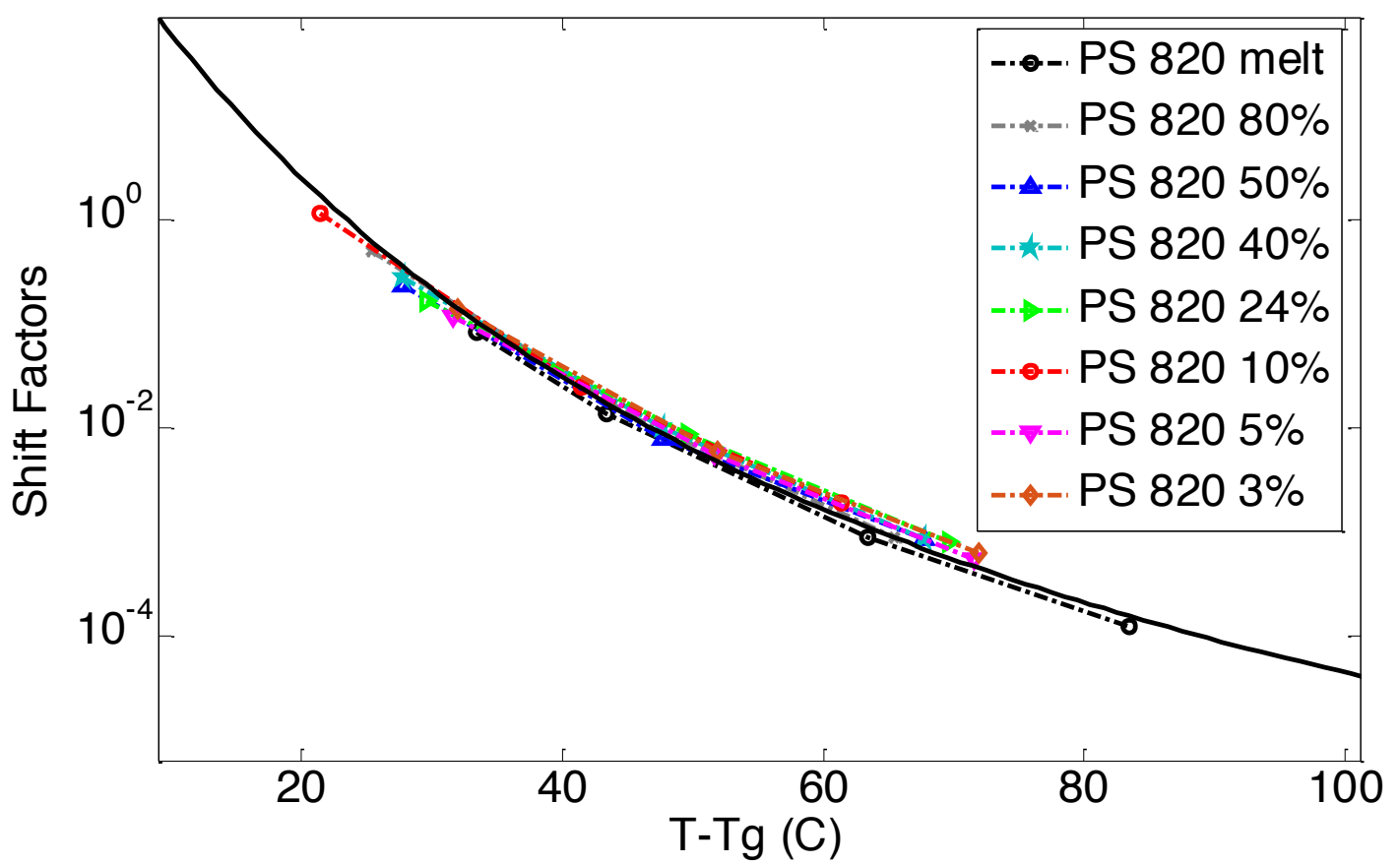

Figure 1: Time Temperature Superposition shift factors as a function of $T-T_{g}$, determined for the different polymers solutions and melt. The continuous curve represents the WLF equation used in ref. [30].

As it can be seen, when plotted in such a way, the values used for PS solutions and the melt nicely coincide. Also, their T-dependence is very close to the dependence found in ref. [30] for the other PS samples described in Table 1, which is represented in Figure 1 by the Williams-Landel-Ferry (WLF) equation ${ }^{35}$. 
$\log a_{T}=\frac{-c_{1}^{0}\left(T-T_{r e f}\right)}{c_{2}^{0}+\left(T-T_{r e f}\right)}$

with $c_{1}^{0}=8.99, c_{2}^{0}=81.53 \mathrm{~K}, \mathrm{~T}_{\mathrm{ref}}=130^{\circ} \mathrm{C}$ and $\mathrm{T}$, the temperature expressed in ${ }^{0} \mathrm{C}$.

The fact that all these curves superimpose also validates the factors used for shifting the data at iso- $\mathrm{T}_{\mathrm{g}}$ condition. Indeed, these shift factors are shown in Figure 1 at $\mathrm{T}=130^{\circ} \mathrm{C}$, i.e. at (T$\mathrm{T}_{\mathrm{g}}$ ) ranging from $23.4^{0} \mathrm{C}$ (for PS820) to $35.4^{\circ} \mathrm{C}$ (for the OS8.8), and they are in good agreement with the WLF equation.

\section{Tube-based Model for entangled linear solutions}

In this Section, the viscoelastic response of the entangled linear solutions is modeled based on the Time Marching Algorithm (TMA) that we have developed, based on the tube theory, for predicting the relaxation modulus of linear and branched entangled polymers ${ }^{37-39}$. The main equations are summarized here below for the specific case of long linear chains of mass $M_{2}$ blended with short chains or oligomers of mass $M_{1}$. Since this approach should also correctly describe the viscoelastic properties of monodisperse chains (of mass $M_{2}$ ) in the melt state, and in order to keep the model as general as possible, the equations are described as a function of the reference material parameters $M_{e, 0, \text { melt }} G_{\mathrm{N}, \text { melt }}^{0}$, and $\tau_{e, 0, \text { melt }}$ of the melt state, and short-long entanglements are considered at very short times even for PS/oligo-S blends.

As mentioned in the Introduction, this approach considers the existence of two length and time scales, the first one being related to the motions of the long chains in their thin (initial) tube, while the second one is related to the motions of the long chains in their dilated tube. Since this second relaxation takes place at the rhythm of the motions of the short chains, i.e. $\tau_{\text {short-long }}$ (see Eq. 7 ), the necessary time to relax one long-long entanglement segment by tension is usually delayed compared to its intrinsic Rouse time, $\frac{\tau_{e 0, m e l t}}{v_{2}^{2 \alpha}}$. Within this framework, using oligomers as short component represents an extreme case: the relaxation of the long chains in a thin tube (i.e. the first length scale) has a negligible effect since it is always slower than their relaxation in the dilated tube (i.e. the second length scale), this last process being not delayed by the motion of the short chains. Thus, in this specific case, the necessary time to relax one long-long entanglement segment is simply described by its 
minimum intrinsic value, $\frac{\tau_{e 0, \text { melt }}}{v_{2}{ }^{2 \alpha}}$ (see Eq. 8$)^{11}$ and the short-long entanglements do not play any role in the relaxation of the long chains, despite the fact that it is considered that they exist at time $t=0$.

Following ref. [11], since the short chains only relax by Rouse process, the general expression of the relaxation modulus can be described as:

$G(t)=\left(v_{1} G_{\mathrm{R}, 1}(t)+v_{2} G_{\mathrm{R}, 2}(t)\right)+G_{d}(t)$.

The first term of this equation describes the full relaxation of the short, unentangled chains by the intrinsic Rouse process ${ }^{41}$ :

$G_{\mathrm{R}, 1}(t)=\frac{\rho_{0} R T}{M_{1}} \sum_{p=1}^{n} \exp \left(-\frac{2 p^{2} t}{\tau_{\mathrm{R}}\left(M_{1}\right)}\right)$

with $\tau_{R}\left(M_{1}\right)$, the Rouse time of the short chains, while the second term describes the fast Rouse relaxation of the long chains, taking place in all directions for molecular sub-chains shorter than the average molar mass between two entanglements, $M_{e, 0, \text { melt }}$ and along the tube axis (longitudinal modes) for the longer modes ${ }^{4}$ :

$G_{\mathrm{R}, 2}(t)=\frac{\rho_{0} R T}{M_{2}}\left\{\frac{1}{4} \sum_{p=1}^{Z_{2,0}} \exp \left(-\frac{p^{2} t}{\tau_{\mathrm{R}}\left(M_{2}\right)}\right)+\sum_{p=Z_{2,0}+1}^{n} \exp \left(-\frac{2 p^{2} t}{\tau_{\mathrm{R}}\left(M_{2}\right)}\right)\right\}$,

with $Z_{2,0}=M_{2} / M_{e 0, \text { melt }}$, the number of entanglement segments of a long chains in the melt state.

In addition to this Rouse relaxation, the chains must disentangle in order to reach their full relaxation state. This is described by the term $G_{\mathrm{d}}(t)$ :

$G_{\mathrm{d}}(t)=G_{\mathrm{N}, \text { melt }}^{0} \varphi^{\prime}(t)\{\phi(t)\}^{\alpha}$, 
with, in this case, the survival fraction of initial (thin) tube at time $t, \varphi^{\prime}(t)$, which only accounts for the unrelaxed segments of the long component: $\varphi^{\prime}(t)=v_{2} \varphi_{2}{ }^{\prime}(t)$ (since the short chains are fully relaxing by Rouse dynamics), and with $\phi(t)^{-\alpha}$ representing the number of initial entanglement segments per dilated segment. While $\phi(t)$ is often equal to $\varphi^{\prime}(t)$, this is not the case here due to the very fast relaxation of the short chains, and it is therefore important to consider that $\phi(t)$ cannot decrease faster than by a Constraint Release Rouse process $(\mathrm{CRR})^{2,11,42}$ :

$$
\phi\left(t_{i}\right)=\max \left\{\varphi^{\prime}\left(t_{i}\right), \phi\left(t_{i-1}\right)\left[\frac{\left(t_{i-1}\right)}{t_{i}}\right]^{\frac{1}{2}}\right\},
$$

with $t_{i-1}$ representing the time step before the time $t_{i}$ and with the initial condition $\left(\phi\left(t=\tau_{e}\right)=1\right) .{ }^{47}$ Thus, this CRR condition prevents the function $\phi(t)$ to decrease faster than with a slope of $-1 / 2$. In fact this is crucial here since it will ensure that the long chains will relax by Rouse dynamics from their initial, undiluted tube $(\phi=1)$ to their dilated tube, corresponding to $\left(\phi=v_{2}\right)$. This Rouse relaxation makes sense since the short chains are oligomers and therefore the undiluted tube does not really exist.

For such extreme blends, the contour length fluctuation of their initial, undiluted, tube (i.e. including the short-long entanglements) can be neglected. Therefore, the survival proportion $\varphi_{2}^{\prime}(t)$ of initial tube segments of the long chains at time $t$, can be described by considering only their relaxation by reptation and their relaxation by tension equilibration process or constraint release activated fluctuations $(\mathrm{CR}-\mathrm{CLF})^{11,37}$ :

$$
\varphi_{2}^{\prime}(t)=\int_{0}^{1} p_{\text {rept }}(x, t) p_{C R-C L F}(x, t) d x
$$

with $p_{\text {rept }}(x, t)$ and $p_{C R-C L F}(x, t)$, the survival probabilities of a chain segment $x$ at time $t$, considering the chain relaxation either by reptation or by CR-CLF. These probabilities depend on the reptation and CR-CLF times of the long chains ${ }^{11,13}$ and are approximated by single exponential functions which compare the time $t$ to these relaxation times, for each segment $\mathrm{x}$. Since the relaxation by tension equilibration is a Rouse process along the axis of the dilated tube, the corresponding relaxation times are well described by the Constraint-Release activated fluctuations process (CR-CLF) taking place in the dilated tube: 
$\tau_{\mathrm{CR}-\mathrm{CLF}}(x)=\left(\tau_{\text {long-long, eql. }}\right) \frac{9 \pi^{3}}{16}\left(v_{2} Z_{2,0} / 2\right)^{4} x^{4}$

with $v_{2}$ the weight fraction of the long chains, $x$ the normalized coordinate of a chain segment, ranging from 0 at the extremities to 1 at the middle of the chain, and $Z_{2,0}$ as the number of entanglement segments along the long chain at time 0 (i.e., considering both long-long and long-short entanglements). As already mentioned, for long chains diluted in oligomers, the Rouse time of a long-long entanglement segment, $\tau_{\text {long-long,eql }}$, is well described by Eq. 8. On the other hand, according to the Struglinsky-Graessley criterion, reptation is taking place in a dilated tube ${ }^{40}$ :

$\tau_{\text {rept }}\left(M_{2}\right)=3 \tau_{e, 0} Z_{2,0}{ }^{3} v_{2}^{\alpha}$

\section{Results and Discussion}

\section{IV.1. Comparison between experimental and theoretical viscoelastic data}

First, the viscoelastic properties of monodisperse entangled PS samples in the melt state (see Table 1) are modelled, based on the approach presented in Section III. This requires defining the values of the three materials parameters corresponding to the melt state, $G_{N, \text { melt }}^{0}, M_{e 0, \text { melt }}$

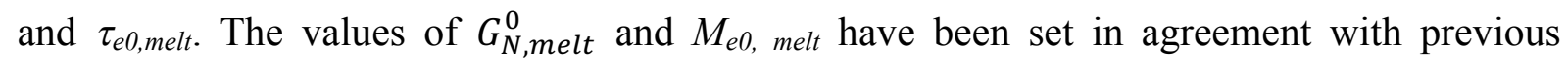
works $^{37,38,43}$ as $230 \mathrm{kPa}$ and $15 \mathrm{~kg} / \mathrm{mol}$ respectively, while $\tau_{e 0, \text { melt }}$ has been fixed to $0.55 \mathrm{~s}$ at the reference temperature of $130^{\circ} \mathrm{C}$. Comparison between experimental and predicted results is shown in Figure 2. A good agreement is found, which validates the values taken for these parameters, as well as the experimental data, which come from three different works and two different research groups. 


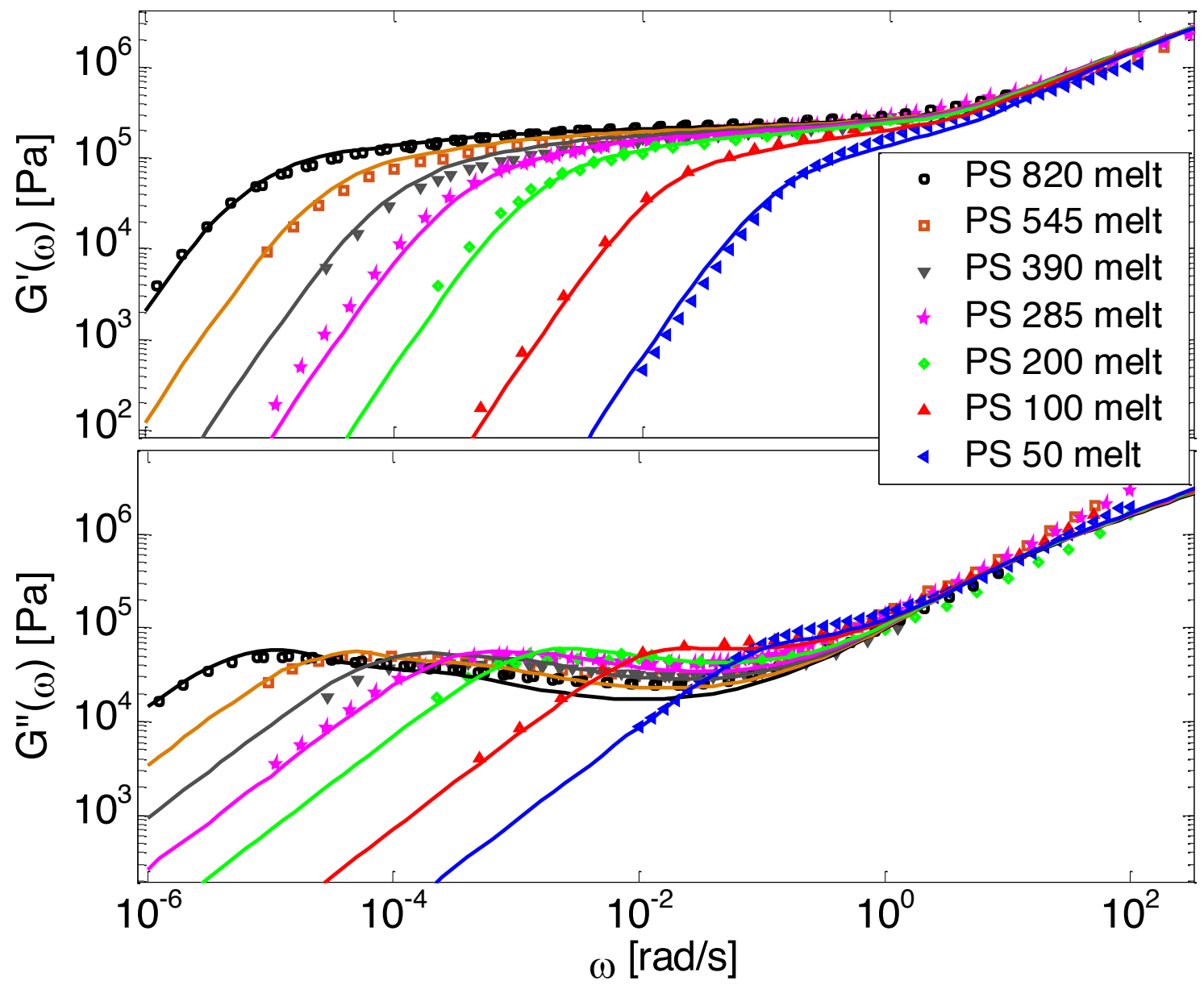

Figure 2: Experimental (symbols) and predicted (line) storage and loss moduli of monodisperse PS samples in the melt state at a reference temperature of $130^{\circ} \mathrm{C}$, with $M_{w}=$ (from left to right:) 820 $\mathrm{kg} / \mathrm{mol}, 545 \mathrm{~kg} / \mathrm{mol}, 390 \mathrm{~kg} / \mathrm{mol}, 285 \mathrm{~kg} / \mathrm{mol}, 200 \mathrm{~kg} / \mathrm{mol}, 100 \mathrm{~kg} / \mathrm{mol}$ and $50 \mathrm{~kg} / \mathrm{mol}$.

The model is then used, without any degree of freedom using only the set melt parameters, to calculate and predict the linear viscoelastic data of the two PS solutions series presented in Table 1. The comparisons between predicted and experimental data are shown in Figures 3 and 4. While the blends presented in Figure 3 are all based on PS820 and OS8.8, Figure 4 presents viscoelastic data of solutions based on different molecular weights PS diluted in OS4 at different concentrations, but with a similar number of (long-long) entanglement segments, $Z_{2}$. It was shown in ref. [30] that the G',G', curves of these different solutions superimpose very well if horizontally and vertically shifted.

One can observe that the approach proposed here allows obtaining very good predictions for different masses and concentrations, spanning the whole range from concentrated and semi- 
dilute solutions down to only few entanglements per chain. In particular, it should be noted that this good agreement of the different plateau moduli between experiment and calculation is found when the actual dilution exponent $\alpha$ has been fixed to $\alpha=1$ in the model.

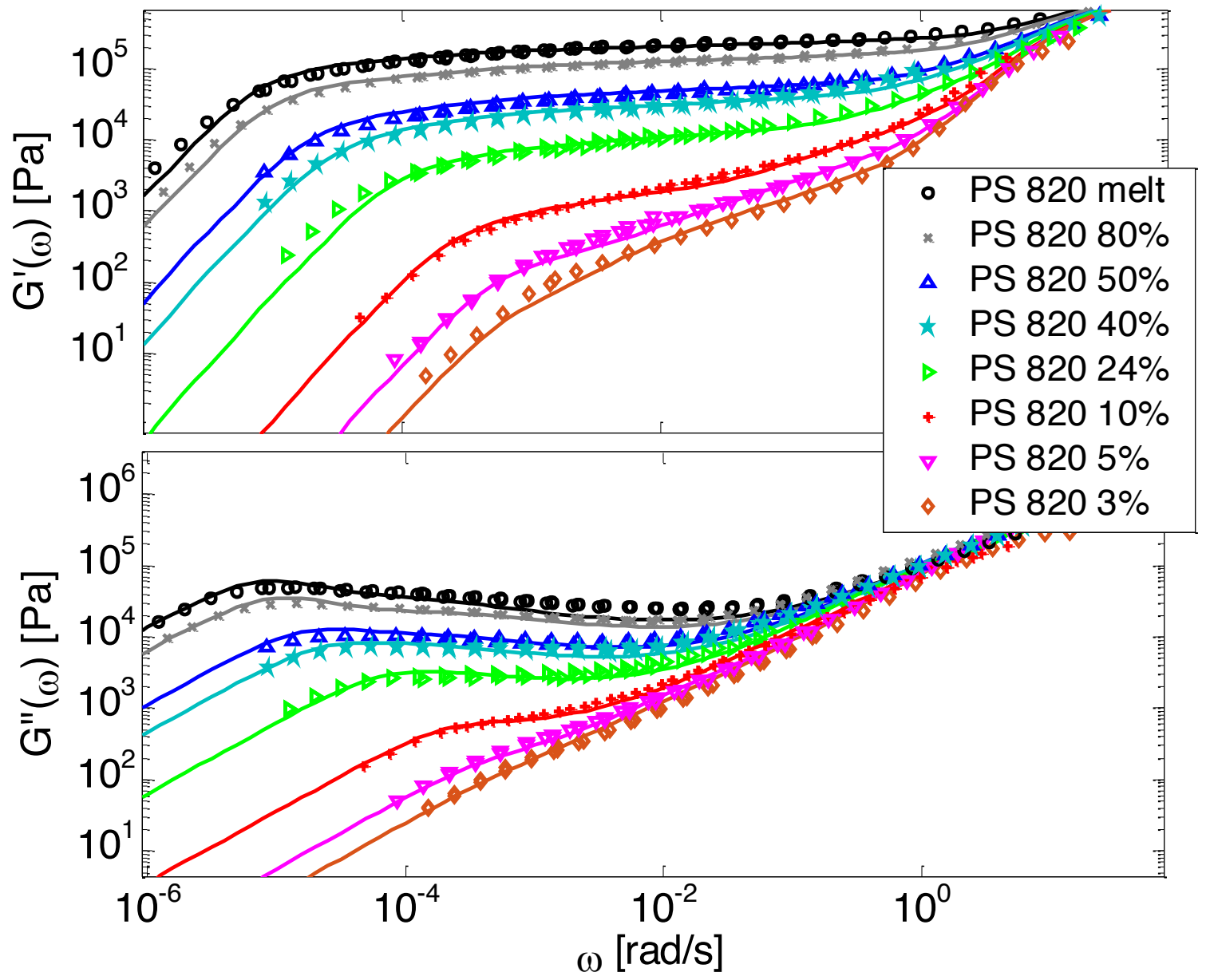

Figure 3: Experimental (symbols) and predicted (line) storage and loss moduli of monodisperse PS sample PS820 diluted in oligomeric styrene OS8.8, at different concentrations. The experimental data are shifted to iso- $T_{g}$ condition. 


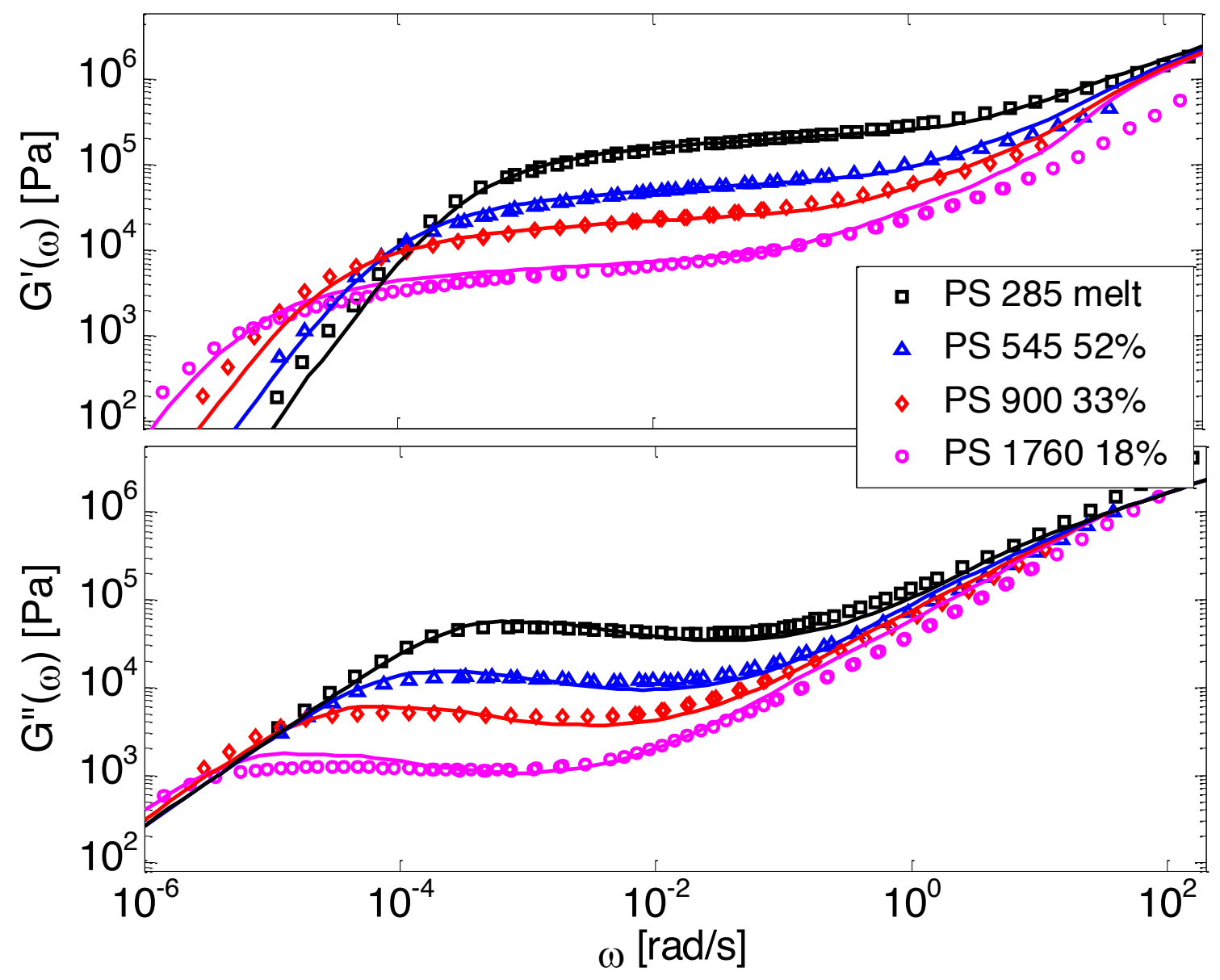

Figure 4: Experimental (symbols) and predicted (line) storage and loss moduli of monodisperse PS samples in the melt state or in solution in the styrene oligomers OS4, at different concentrations. The experimental data are shifted to iso- $T_{g}$ condition.

Based on these results, several observations can be made. First, one can observe in Figure 3 that the shape of the viscoelastic data at intermediate and high frequencies is rather complex. In particular, when the long chains are strongly diluted, we observe the appearance of different slopes in this frequency region. This can be attributed to the fact that these curves include both the relaxation of the short component (with $\mathrm{M}_{\mathrm{w}}=8.8 \mathrm{~kg} / \mathrm{mol}$ ) and the Rouse relaxation of the long chains up to molecular segments of mass $M_{e, 0, \text { melt }} / v_{2}$ (see Eq. 14). This is illustrated in Figure 5, which presents the predicted storage and loss moduli of sample PS820 diluted at $5 \mathrm{wt} \%$ in OS8.8, and shows the specific contributions of the different relaxation processes to the sample relaxation. 


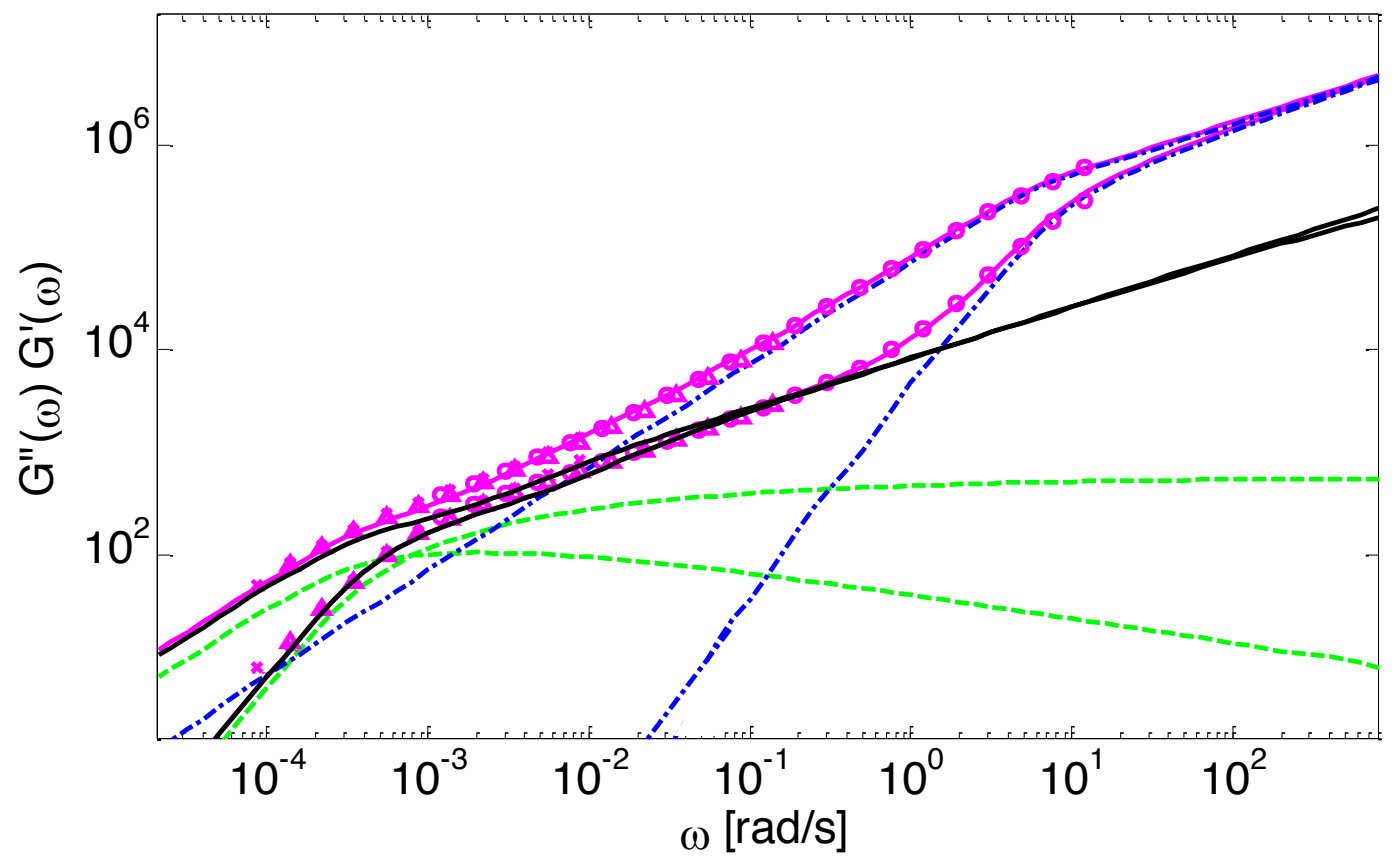

Figure 5: Experimental (symbols) and predicted (lines) storage and loss moduli of the sample PS820 diluted to $5 \mathrm{wt} \%$ in OS8.8. The data have been divided into the contribution of the oligomers (blue dash dot curves) and of the long chains, including the Rouse and Constraint Release Rouse modes (black curves) or neglecting them (green dashed curves).

As it can be seen, the curves observed in the frequency window between 1 and $20 \mathrm{rad} / \mathrm{s}$ correspond to a transition state between two Rouse domains with typical slopes of $1 \frac{1}{2}$ : The first (high frequency) process includes the relaxation of both the short and the long chains and corresponds to the fast modes of Rouse, i.e. the Rouse relaxation of polymer strands shorter than $M_{l}$, while the second Rouse relaxation only involves the longest modes of the long chains and is associated with the relaxation of polymer strands of masses between $M_{1}$ and $M_{e}=M_{e, 0, \text { melt }} / v_{2}$. The existence of this second frequency domain governed by Rouse relaxation shows the importance of accounting for the Constraint Release Rouse modes of the long chains, taking place up to the long-long entanglement segments of mass $M_{e}$ in order to properly capture the relaxation behavior of such blends with large difference in $M_{w}$. Though this might be obvious for the case of polymer solutions, this point becomes more delicate for binary blends of short and long molecules. Indeed, as described in Section III, this Rouse-like relaxation, which allows the long chains to move from a thin tube to a dilated tube, is usually described by the Constraint Release Rouse process (CRR) through Eq. $14 .{ }^{11}$ However, this requires to accurately define the relaxation state of the chains at the time the CRR becomes active. While it is easy to determine this state in case of long chains diluted in oligomers (by 
imposing $\left.\phi\left(t=\tau_{e}\right)=1\right)$, for longer short chains the starting time of CRR and the corresponding relaxation state are not known a priori.

In Figure 5, one can also observe that the rubbery plateau is quite low and not well defined. This can be understood based on the fact that when diluted to $5 \mathrm{wt} \%$, sample PS 820 only contains an average of 2.7 entanglement segments per chain, which is at the limit of the entangled state. In the terminal zone, the chains are therefore moving by CR-CLF in a very dilated tube. In order to observe an actual (rubbery) plateau, the concentration of sample PS820 must be at least $10 \mathrm{wt} \%$ (see Figure 3).

As seen in Figure 2 for the monodisperse samples, the high frequency data are not accurately captured by the model. This is due to the contribution of the terminal tail of glassy relaxation ${ }^{44}$, which is not included in the model. On the other hand, the discrepancy observed in Figure 4 at high frequency has two different origins: in addition to be effected by the glassy relaxation $^{44}$, large part of the disagreement comes from the fact that our model predicts slower Rouse relaxation of the oligomers when compared to the experimental results. This cannot be corrected by considering another value for the material parameter $\tau_{e 0, \text { melt }}$ (used to define the intrinsic Rouse time of the oligomers) since this would strongly affect the other data. However, since this discrepancy is observed only at very high frequency, it does not influence the curves around the rubbery plateau, i.e. in the frequency area where we would like to discuss the results and the value of the effective dilution exponent.

The good agreement found between experimental and theoretical curves allows us to take advantage of the model for further investigating how the effective (i.e. experimentally determined from the scaling of $G_{\delta}$ or $G{ }^{\prime \prime}{ }_{\max }$ with $c$ ) value of $\alpha$ varies with the sample composition. This is the objective of the next Section.

\section{IV.2. Influence of concentration on $G_{N}^{0}, G_{\delta}, G^{\prime}{ }_{\max }$ and on the effective $\alpha$}

As already mentioned, the plateau modulus of the different blends in Figures 2-4 is well described by the model in which the actual dilution exponent is fixed to the a priori expected value of $\alpha=1$. This good agreement between theoretical and experimental values means that the real plateau modulus of these samples, $G_{N}^{0}$, must scale with $v_{2}^{2}$, or equivalently, with $c^{2}$ 
(see Eq. 3). However, due to the CR-CLF (tension equilibration) process, the observable level of the plateau measured at lower frequency, is expected to change so that the effective value of $\alpha$, measured at longer times, is expected to be larger than 1 . It is therefore interesting to determine its value for the sets of samples analyzed here, based on approaches usually used in literature in the case of monodisperse samples. Such approaches are the determination of the effective $\alpha$ by looking at the c-dependence of the maximum loss modulus, $G$ " ${ }_{\max }$, localized in the terminal regime ${ }^{14,17,18}$. On the other hand, in order to estimate the influence of the tension equilibration at intermediate frequency regime, another approach is to look at the $c$ dependence of $G_{\delta}$, defined as the value of the storage modulus at the frequency $\omega_{\delta}$ where the $\tan \delta$ curve ( $=G$ " $/ G^{\prime}$ ) shows a minimum. ${ }^{45,46} G_{\delta}$ is usually used to approximate the value of the plateau modulus $G_{N}^{0}$.

Results obtained for the two sets of solutions are shown in Figure 6. The theoretical $G_{\delta}$ values have also been determined and are represented by the cross symbols in Figure 6.a.
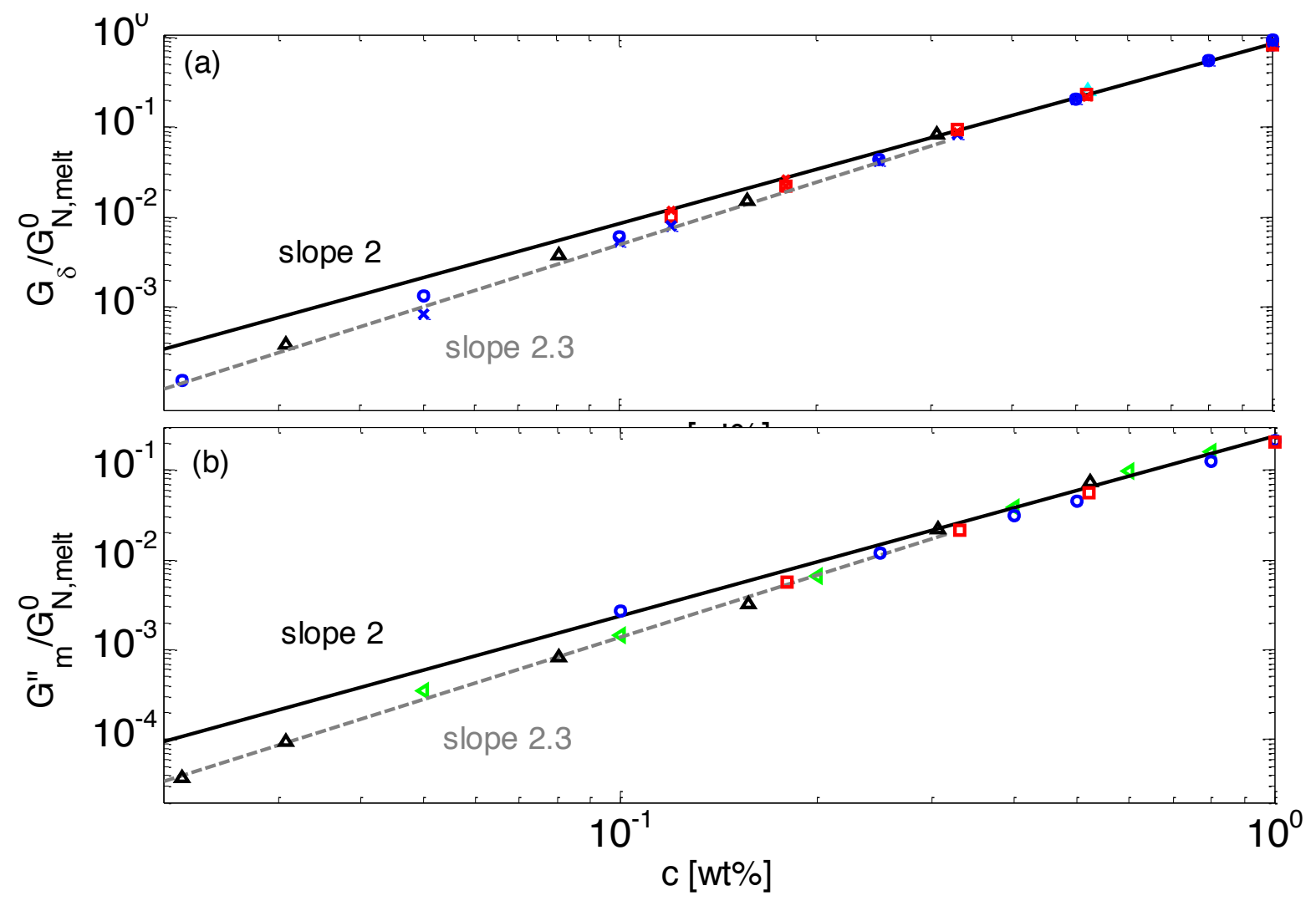

Figure 6: Values of (a) $G_{\delta}$, and (b) $G{ }^{\prime}{ }_{\text {max }}$ determined from the experimental $(\square, o, \Delta,<)$ or the predicted $(X)$ data of the blends. The data correspond to PS/OS blends with similar number of 
entanglements (Red $\square$ ), the PS820/OS8.8 blends (blue o), the PBD410/PBD3 blends (green $<$ ) and the PBD925 diluted in small-molecule solvent (black $\Delta$ ) (see Table 1). Solid and dashed line represent the slope of 2 and 2.3. The line in Figure (b) are plotted a factor 3.56 times lower than in Figure (a).

As a first comment, it must be noted that $G{ }^{\prime}{ }_{\max }$ cannot be determined for low concentrations of PS in oligomers since there is no clear maximum in the corresponding loss modulus curves, due to the overlap between constraint release Rouse process and terminal relaxation of the chains (see Figure 5). Only the plateau of the PBD samples could be measured, the long chains being much more entangled than the PS long chains. On the other hand, $G_{\delta}$ could be measured only for the samples with experimental data in the intermediate-high frequency region.

Figures 6 reveals important features:

- From the comparison between $G{ }^{\prime}{ }_{\max }$ and $G_{\delta}$, we can see that the $G{ }^{\prime}{ }_{\max }$ values are indeed around a factor 3.56 lower than the values of $G_{\delta}{ }^{18}$ Also, we observe that the c-dependence of $G^{\prime \prime}{ }_{\max }$ and $G_{\delta}$, are very similar and that they both differ from the real plateau modulus $G_{N}^{0}$ (represented by the continues line in Figure 6.a), this discrepancy increasing with decreasing polymer concentration. From this result, we conclude that the CR-CLF process can already affect the viscoelastic properties at the intermediate frequency $\omega_{\delta}$. Thus, the $G_{\delta}$, obtained from the $\tan \delta$ method cannot be used to determine $G_{N}^{0}$ for polymer/oligomers blends since it is already influenced by the fast tension equilibration process.

We also see that the $G{ }^{\prime}{ }_{\max }$ data are a little more scattered than the $G_{\delta}$ data. This is most probably due to the fact that $G{ }^{\prime}{ }_{\max }$ is much more sensitive to polydispersity than $G_{\delta}$.

- At high concentrations ( $>40 \mathrm{wt} \%), G{ }^{\prime}{ }_{\text {max }}$ and $G_{\delta}$, show a c ${ }^{2}$-dependence, which corresponds to an effective value of $\alpha$ equal to 1 . Then, at lower concentration, most of the blends show a transition from $\mathrm{c}^{2}-$ to $\mathrm{c}^{2.3}$ - dependence of their plateau values. This demonstrates that the value of the effective $\alpha$ determined from either $G^{\prime}{ }_{\max }$ or $G_{\delta}$, is not a constant and depends on the concentration.

- Looking at the $G_{\delta}$ extracted from both the experimental and the theoretical curves (see Figure 6.a), it is interesting to note that the set of PS/OS4 blends prepared from different molecular weight PS samples in order to keep $Z_{2}$ constant (set 2, see the red symbols), keeps a 
$\mathrm{c}^{2}$-dependence also at lower concentrations, while this is not the case for the other blends, made from long chains with a specific, constant molar mass and varying concentrations. In particular, the value of $G_{\delta}$ of PS3200 diluted to $13 \mathrm{wt} \%$ is well above the $G_{\delta}$ found for a solution of $13 \mathrm{wt} \%$ of PS820. From this observation, one can conclude that the effective $\alpha$ determined from $G_{\delta}$ (at intermediate frequency) is not only a function of concentration, but also seems to depend on the molar mass of the chain.

This is further illustrated in Figure 7, which compares the viscoelastic response of two solutions of PS chains, both at the same concentration of $10 \mathrm{wt} \%$, but with chain molar masses of either $820 \mathrm{~kg} / \mathrm{mol}$ or $3900 \mathrm{~kg} / \mathrm{mol}^{34}$ : despite the fact that they are diluted to the same concentration and thus have the same entanglements density, it is obvious that the value of $G_{\delta}$, is much lower for sample PS820 (which contains 6 long-long entanglements) in comparison to the $G_{\delta}$, value of the PS3900 sample (which contains around 28 long-long entanglements).

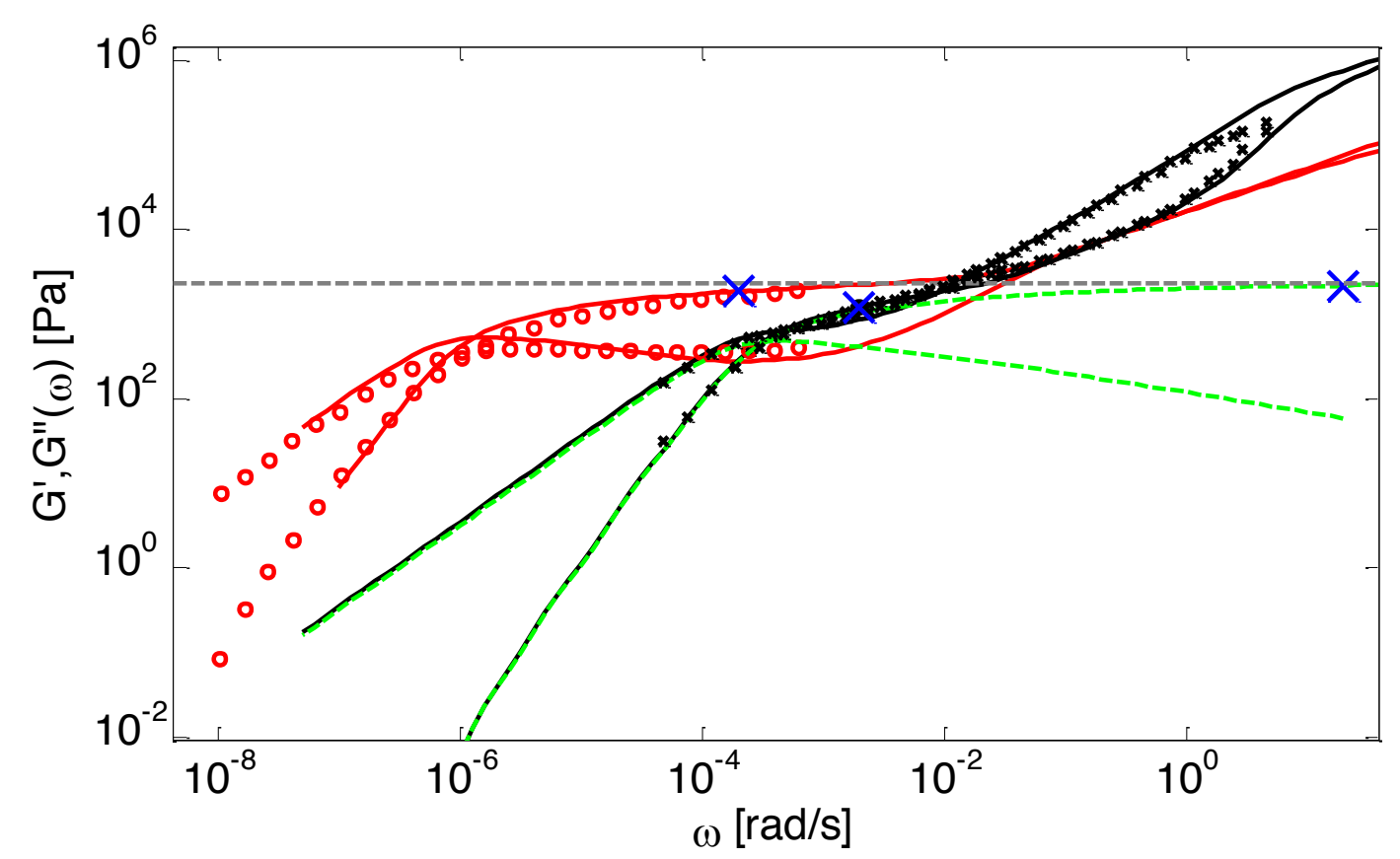

Figure 7: Experimental (symbols) and predicted (line) storage and loss moduli of monodisperse PS samples in solutions, for sample PS820 diluted to $10 \mathrm{wt} \%$ (black $x$ ) and for sample PS3900 diluted to $10 \mathrm{wt} \%(\mathrm{red}, \mathrm{o})$. The $X$ symbols on the predicted curves indicate the storage modulus which corresponds to the minimum of the tan $\delta$ curve. The predicted curves of PS820 are shown with (black 
lines) and without (green lines) Rouse contributions. The dashed line corresponds to the rubbery plateau (based on $\alpha=1$ ).

From these observations, a global picture can be drawn: while the effective value of $\alpha$ in binary blends of long and short linear chains must be determined from moduli in the frequency range close to the terminal relaxation of the long chains ${ }^{11}$, for polymer/oligomers blends a larger effective $\alpha$ can also be determined from moduli at higher frequencies, with no significant difference between the c-dependence of the values of $G{ }^{\prime}{ }_{\max }$ and $G_{\delta}$. This is due to the fact that, in the case of polymer/oligomer blends, the tension equilibration process, which is modelled here by the CR-CLF process, is very fast since it does not include any delay due to the motions of the short component (see Equations 7 and 8), and the long chains can explore their dilated tube as fast as their intrinsic Rouse process allows. Since these fast Rouse motions are already taking place at times shorter than the inverse of the frequency $\omega_{\delta}$ at which $G_{\delta}$ is determined, the effective $\alpha$ at intermediate frequency is found to be larger than 1.

If we consider that a large value of the effective $\alpha$ is related to the tension equilibration process, as it is suggested in this work, one can easily explain that its value can vary between 1 (no influence of the CR-CLF) and 4/3 (fully equilibrated long chains ${ }^{17}$ ), depending on both concentration and molar mass of the long chains. Indeed, from Equation 16 it is expected that the importance of the CR-CLF process increases with decreasing chain length or the concentration, i.e. the effective $\alpha$ increases with a decreasing number of long-long entanglements per chain, $Z_{2} \cdot{ }^{47}$. This $Z_{2}$ dependence of the effective $\alpha$ is consistent with the $\mathrm{c}^{2}$ dependence found for $G_{\delta}$ with the second set of PS-OS blends, for which $Z_{2}$ was kept constant for all concentrations ${ }^{30-31}$ : in such a case the importance of CR-CLF process is exactly the same for all the samples since they all have a constant number of entanglements. Thus, they are all characterized by a similar effective $\alpha$ and the slope of the $G_{\delta}$ values continues to scale with $c^{2}$. On the contrary, the dependence of $G_{\delta}$ for the PS820/OS8.8 blends shows a power law which is relatively well defined by a slope of $7 / 3$ at low concentrations: by diluting PS 820 in oligomers, the number of (long-long) entanglement segments is decreasing; this enhances the CR-CLF process, which is already observed at intermediate frequency since it is not 
delayed by the short chains. As a consequence, the difference between $G_{\delta}$ and $G_{N}^{0}$ is increasing when the PS820 is diluted in the oligomers.

Thus, the tension equilibration process allows us to explain the non-constant value of the effective $\alpha$ found experimentally from $G{ }^{\prime \prime}{ }_{\max }$ and $G_{\delta}$, as well as its dependence on concentration and molar mass. According to the CR-CLF process, its value should depend on $Z_{2}$, which is further validated in Section IV.3.

Another consequence of this fast CR-CLF process is that for such blends, $G_{\delta}$ cannot be considered as a good approximation of the plateau modulus $G_{N}^{0}$, which scales with $\mathrm{c}^{2}$ for all concentrations (as defined in our model). This is illustrated in Figure 7, which shows the storage modulus corresponding to $G_{\delta}$ for the two blends, and compares this value to the real plateau modulus $G_{N}^{0}$ determined by removing the contribution of the high frequency Rouse relaxation as well as the CR-Rouse modes (see Eq. 14) from the theoretical curves ${ }^{46-48}$. It is seen that, due to the overlap between Rouse relaxation and CR-CLF process, the minimum of the $\tan \delta$ curve is found at a rather low frequency $\omega_{\delta}$ (see the $\mathrm{X}$ symbols), at which the sample has already partially relaxed by tension equilibration.

\section{IV.3. Influence of $Z_{2}$ on the terminal relaxation time, CR-CLF process and effective $\alpha$.}

As described in Section IV.2., it seems that the value of the effective $\alpha$ depends on the importance of the CR-CLF process: the more the chains are able to relax by CR-CLF before their terminal relaxation, the larger is the effective $\alpha$, which is usually determined based on the scaling of $G{ }^{\prime \prime}{ }_{\max }$ with concentration, but which can also be determined from $G_{\delta}$ in the specific case of polymer/oligomers blends. Thus the transition between an effective $\alpha$ of 1 (negligible effect of CR-CLF) and 4/3 (fully equilibrated chains ${ }^{17}$ ) could, in principle, take place at any possible concentration, depending on the entanglement state of the long chains, $\mathrm{Z}_{2}$. In this Section, we first try to point out the existence and importance of the CR-CLF process as a function of $Z_{2}$, based on the experimental terminal relaxation time of the solutions, and then relate this process to the effective $\alpha$ determined from $G_{\delta}$. 
As mentioned in the Introduction, the terminal relaxation time of the solutions is expected to be a function of both reptation and CR-CLF processes. Since the real dynamic dilution exponent $\alpha$ is equal to 1 , the reptation time of the monodisperse long chains diluted in oligomers (or theta solvent) should therefore scale with concentration (see Eq. 17): $\tau_{\text {rept }, 0}\left(M_{2}\right)=\tau_{\text {rept } 0, \text { melt }}\left(M_{2}\right)$.c. Therefore, by comparing this theoretical reptation time to the experimental terminal relaxation times of these samples, one should be able to extract the importance and scaling of the CR-CLF process. In order to do so, the experimental terminal relaxation time $\tau_{\text {rel }}$, must be determined. This is achieved by superimposing terminal slopes of 1 and 2 to the experimental loss and storage moduli curves in the terminal regime and by defining $\tau_{r e l}$ as the inverse of the frequency at which these two lines cross each-other ${ }^{3}$.

Terminal and reptation times are compared in Figure 8.a, as a function of the concentration, and in Figure 8.b as a function of the number of long-long entanglement segments, $\mathrm{Z}_{2}$, which leads to several observations:

First, one can clearly see that reptation alone is not enough to explain the relaxation of the samples: as expected, Contour Length Fluctuations (CLF) process (for the monodisperse PS samples) and CR-CLF (for the solutions) are needed in order to explain the shorter terminal relaxation times. Also, the ratio between terminal and reptation relaxation time cannot be seen as a simple function of concentration; it is rather a function of the number of long-long entanglement segments along the long chains, $Z_{2}\left(=Z_{2,0} . c\right)$. Indeed, as shown in Figure 8.b., all the data superimpose when plotted in this way and create a master curve, expect for the PBD925/DOP solutions, which are discussed in Section IV.4.

In Figure 8.b., a transition is observed around $Z_{2}=20$, from a terminal time dominated by reptation at high $Z_{2}$, towards much shorter terminal times at low $Z_{2}$, for which another scaling is observed: the terminal relaxation time of the diluted chains containing less than 20 longlong entanglement segments is found to scale with $\left(Z_{2,0}^{3} c\right) \cdot Z_{2}$, i.e. with $\left(c^{2} Z_{2,0}{ }^{4}\right)$. This scaling is identical to the scaling of the CR-CLF process (see Eq. 16) and therefore validates this relaxation mechanism and demonstrates its importance for moderately entangled linear chains diluted in oligomers. In fact, for such chains the reptation process (taking place in a tube only formed by the long-long entanglements) is becoming very slow compared to the CR-CLF process, and its influence becomes negligible. 

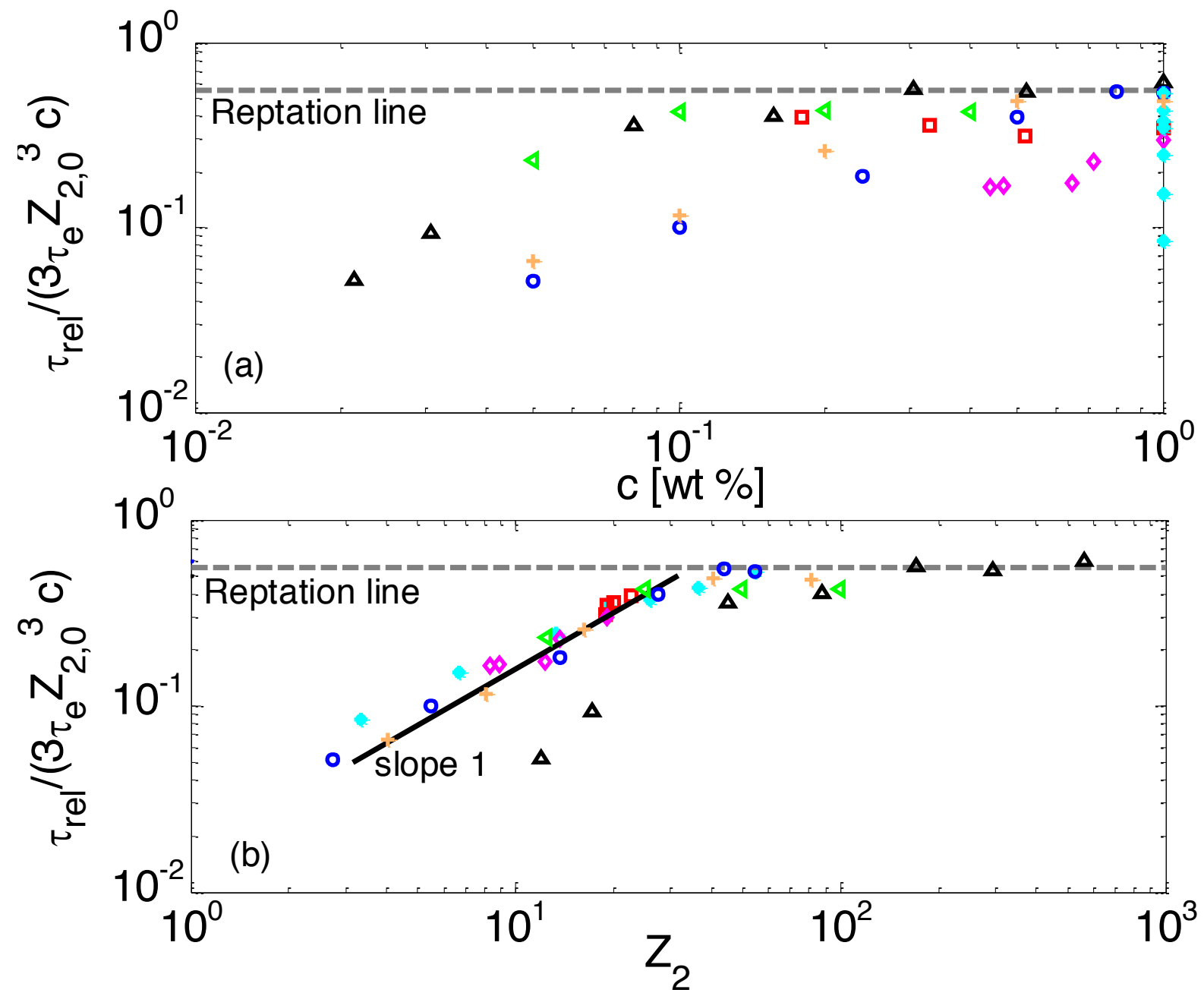

Figure 8: (a) Terminal relaxation time of the long chains normalized by their reptation time in the melt state, $\tau_{\text {rept,omelt }}$ versus the polymer concentration, (b) Terminal relaxation time of the long chains

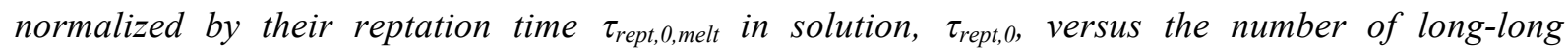
entanglement segments $Z_{2}=Z_{2,0} . c$, for the set of samples with similar number of entanglements (red 口), the PS820/OS8.8 blends (blue o), the PBD410/PBD3 blends (green <), PI308kg/mol/PI2kg/mol blends from ref. [15] (orange +), the PS285kg/mol/OS4kg/mol from ref. [49] (magenta $\diamond)$, the monodisperse PS samples (cyan *) and the PBD925 diluted in small-molecule solvent (black $\triangle$ ) (see Table 1).

Similarly to the terminal relaxation time, the c-dependence of $G_{\delta}$ data can be rationalized when they are plotted as a function of $Z_{2}$. This is illustrated in Figure 9, based on theoretical moduli: the values of $G_{\delta}$ determined from theoretical storage and loss moduli are shown in Figure 9.a. for three different molar masses and for a large range of concentrations, while Figure 9.b. shows the same data but plotted as a function of $Z_{2}$. 

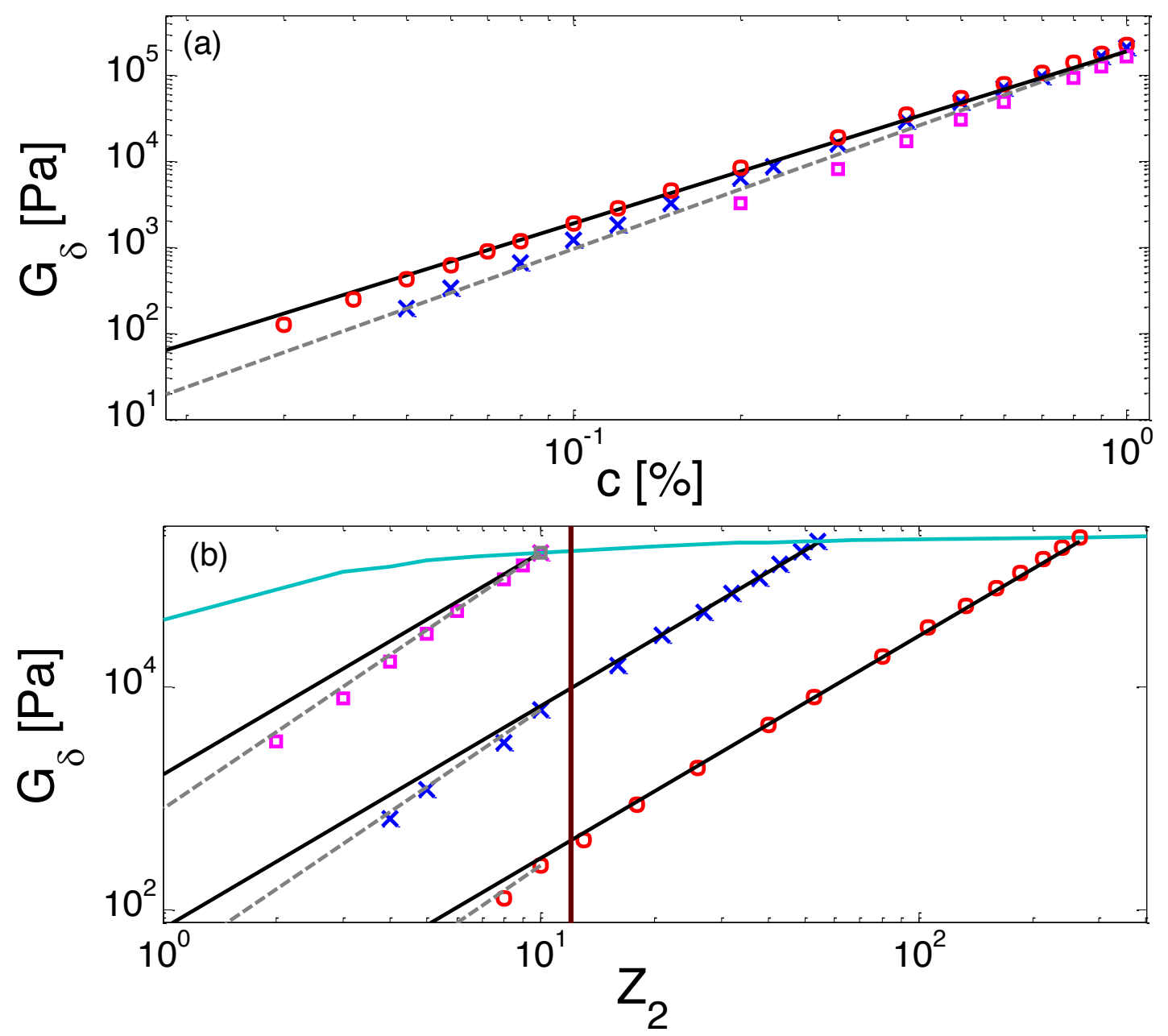

Figure 9: Theoretical predictions of the tan $\delta$ plateau modulus of PS solutions with Mw=4000k (red o), $M w=820 \mathrm{k}$ (blue $x$ ) and $M w=150 \mathrm{k}$ (magenta ) at different concentrations, (a) versus concentration, and (b) versus number of entanglements. The black and grey straight lines have slope of 2 and 2.3 respectively. The light blue curve represents the plateau modulus of monodisperse linear chains.

As it can be seen in Figure 9.a, the c-dependence of $G_{\delta}$ varies with the molar mass of the chains, as it has been observed from the experimental data. Furthermore, it also depends on the range of concentrations which is considered. However, when plotted as a function of $Z_{2}$ (see Figure 9.b.), one can see that, despite the different levels of $G_{\delta}$ (which comes from the different concentrations (see Eq. 3)), all the curves show a transition from an effective $\alpha$ equal to 1 to a larger value at the same number of entanglements, corresponding to around 12 entanglements. While the fact that the effective $\alpha$ tends to $4 / 3$ at low concentrations cannot be determined from the equations, this scaling is clearly observed in Figure 9. 
While the CR-CLF process starts to affect the terminal relaxation time around $Z_{2}=20$, it starts to have a large influence on $G_{\delta}$ only at $Z_{2}=12$. This difference in the transition can be understood by the fact that $G_{\delta}$ is less sensitive to $Z_{2}$ than the CR-CLF process (see Eq. 16). These results suggest that, in order to experimentally determine an effective $\alpha$ equal to 1 from $G{ }^{\prime}{ }_{\max }$ or $G_{\delta}$, the diluted chains should at least conserve 12 (long-long) entanglements. This is in good agreement with the experimental results shown in Figure 6 (apart from the PBD925/DOP solution - see Section IV.4.) as well as with other results found in literature. For example, in ref. [15,16], Watanabe et al. found an effective $\alpha$ of 1.3 for polyisoprene chains of mass of $308 \mathrm{~kg} / \mathrm{mol}$ diluted in oligomers. Indeed, when diluted at $10 \mathrm{wt} \%$, these long chains only contain around 8 long-long entanglements and therefore are already largely influenced by the CR-CLF process. This is also consistent with the results found by Wang et al. [10], who showed that c-dependence of $G{ }^{\prime}{ }_{\text {max }}$ for long polybutadiene chains of $410 \mathrm{~kg} / \mathrm{mol}$ diluted in oligomers at concentrations larger than $5 \%$, i.e. with $Z_{2}>12$, leads to an effective $\alpha$ of 1 (see Fig. 7 in ref. [10]), as well as Tao et al. in ref. [50], who showed that solutions of linear hydrogenated polybutadiene chains with a molar mass of 200, 350 and $440 \mathrm{~kg} / \mathrm{mol}$ and concentrated above $20 \mathrm{wt} \%$ of polymer chains (i.e. with at least 24 entanglements) are well described with an effective $\alpha$ of 1 . Another interesting example has been recently proposed by Park et al., who studied the dilution effect of ultra-high $\mathrm{Mw}$ polyethylene $\left(M_{w}>10^{6} \mathrm{~g} / \mathrm{mol}\right)$ diluted in paraffinic oil ${ }^{51}$. The molar mass of such sample is so high that even at the lowest investigated concentration of $2.5 \mathrm{wt} \%$, the sample shows a clear rubbery plateau. In this work also, it was found that the viscoelastic data of this sample in solutions correspond to an effective $\alpha$ of 1 .

In this part, it has been shown that the chains containing less than 20 long-long entanglements mainly relax by CR-CLF process, and their terminal relaxation time, which is well predicted based on $\alpha=1$, scales with $\left(c^{2} Z_{2,0}{ }^{4}\right)$. The chain equilibration through CR-CLF process leads to a partial relaxation of the long chains and thus to a decrease of the corresponding level of the storage modulus. This effect is a function of $\mathrm{Z}_{2}$, and is already detected at intermediate frequency in the case of polymers/oligomers blends. This larger dilution effect of the oligomers leads to larger value of the effective $\alpha$, especially for blends with $Z_{2}<12$.

\section{IV.4. Solutions in a small-molecule solvent versus polymer/oligomer blends}


From the examples above, we can conclude that the proposed model leads to a unified picture, which is in good agreement with the experimental data. However, while this picture holds for the different polymers/oligomers blends which have been tested, it does not seem to describe the linear viscoelastic properties of entangled polymers strongly diluted in a small-molecule solvent. This is discussed in this Section.

We first focus on the set of solutions proposed by Colby et al. ${ }^{14}$, from which an $\alpha$ value of $4 / 3$ has been obtained. These are solutions of polybutadiene chains of mass $M_{w}=925 \mathrm{~kg} / \mathrm{mol}$ dissolved in dioctyl phthalate (considered as theta solvent). Figure 10 shows the comparison between their experimental and predicted storage modulus. Parameters used in the tube model are the material parameters usually used with the TMA model for polybutadiene samples (i.e. a plateau modulus of $1.2 \mathrm{MPa}, M_{e}=1650 \mathrm{~g} / \mathrm{mol}$ and $\left.\tau_{e}=2.310^{-7} \mathrm{~s}\right)^{38}$. As it can be seen, while the model accurately captures the viscoelastic properties of the higher concentrated solutions (polymer concentration of $100 \mathrm{wt} \%, 52.3 \mathrm{wt} \%$ and $30.6 \mathrm{wt} \%$ ), it starts to deviate at intermediate concentrations (15.7 $\mathrm{wt} \%$ and $8.06 \mathrm{wt} \%)$, and the deviation becomes very large at very low concentration ( $3.08 \mathrm{wt} \%$ and $2.14 \mathrm{wt} \%$ ): it is clear that the level of the predicted plateau modulus is too high compared to the experimental data, despite the fact that the CRCLF process is taken into account. In fact, as discussed in Section IV.3., this last process becomes really effective only if $Z_{2}<12$, while here, $Z_{2}=17$ and 12 for the solutions at 3.08 $\mathrm{wt} \%$ and $2.14 \mathrm{wt} \%$ respectively (based on $\alpha=1$ ).

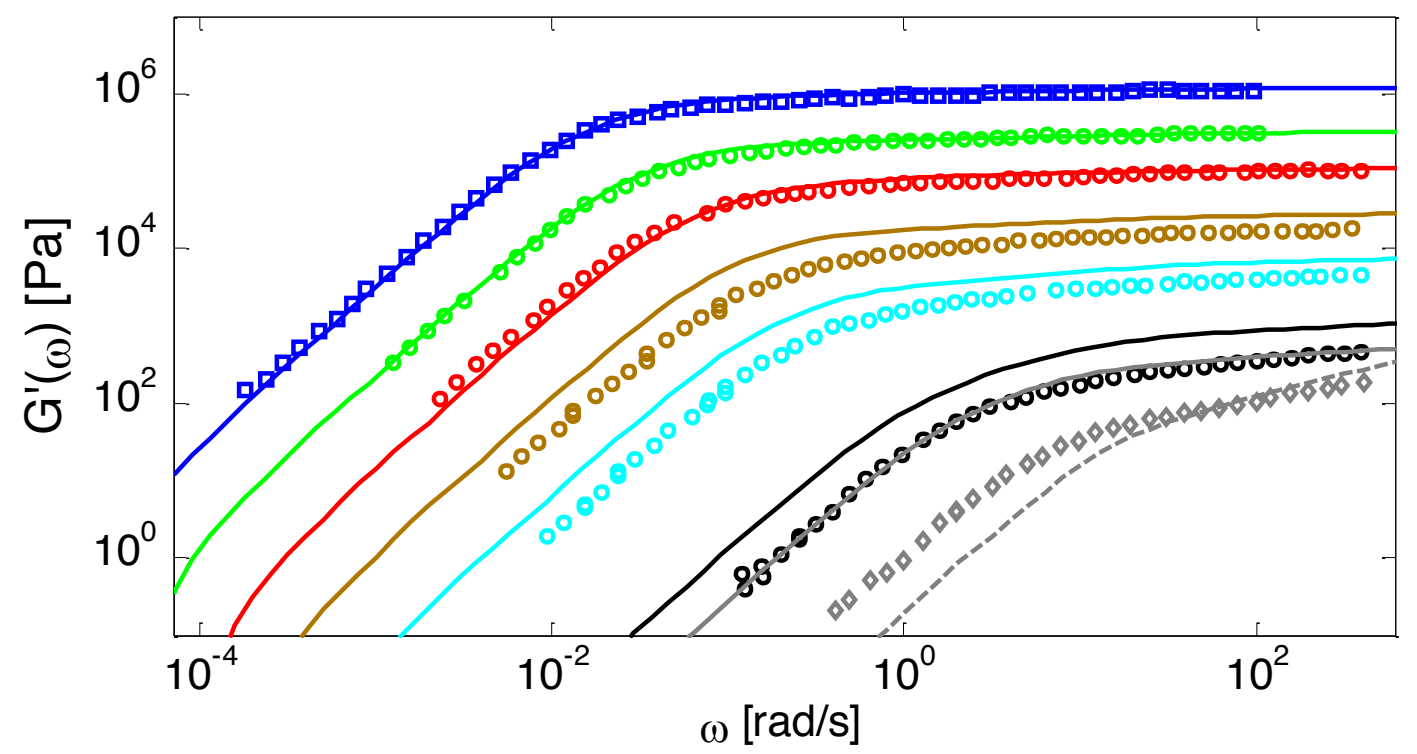


Figure 10: Experimental (o) and predicted (-) storage modulus of PBD925 diluted in DOP at a polymer concentration from $100 \%$ (blue ), to $52.3 \%$ (green), $30.6 \%$ (red), $15.7 \%$ (brawn), $8.06 \%$ (cyan), $3.08 \mathrm{wt} \%$ (black)and $2.14 \%$ (grey $\diamond)$. The dashed curve shows the Rouse relaxation of 2.14 $w t \%$ of PBD925 (i.e. considered as unentangled).

Also, as observed in Figure 10, the sample PBD925 diluted at $2.14 \mathrm{wt} \%$ shows a viscoelastic behavior close to full relaxation by Rouse (see the dashed curve), as if the long chains were nearly not entangled, which definitely does not correspond to the behavior of a chain containing 12 entanglements. Thus, when highly diluted, these PBD chains seem less entangled than the predicted number of entanglements based on $\alpha=1$ and accounting for CRCLF. In such a case, using a larger value of the real dilution exponent $\alpha$ could, indeed, leads to better agreement.

However, considering a larger exponent $\alpha$ would have large consequences for the predictions of the viscoelastic properties of the polymers/oligomers blends, which are well described with $\alpha=1$. We thus face a situation in which our tube model cannot correctly predict the linear viscoelastic properties of both the polymer/oligomers blends and these solutions in a smallmolecule solvent. This is illustrated in Figure 11, which compares a PBD solution of ref. [14], composed of $2.14 \mathrm{wt} \%$ of sample PBD925 in DOP, to the blend composed of $5 \mathrm{wt} \%$ of PBD410 in PBD3 ${ }^{10}$ : although of different composition, these two samples have a similar number of entanglements $\left(Z_{2}=12\right.$ and 12.4 respectively) if we consider $\alpha=1$ (by considering $\alpha=1.3, Z_{2}=3.8$ and 5 respectively).

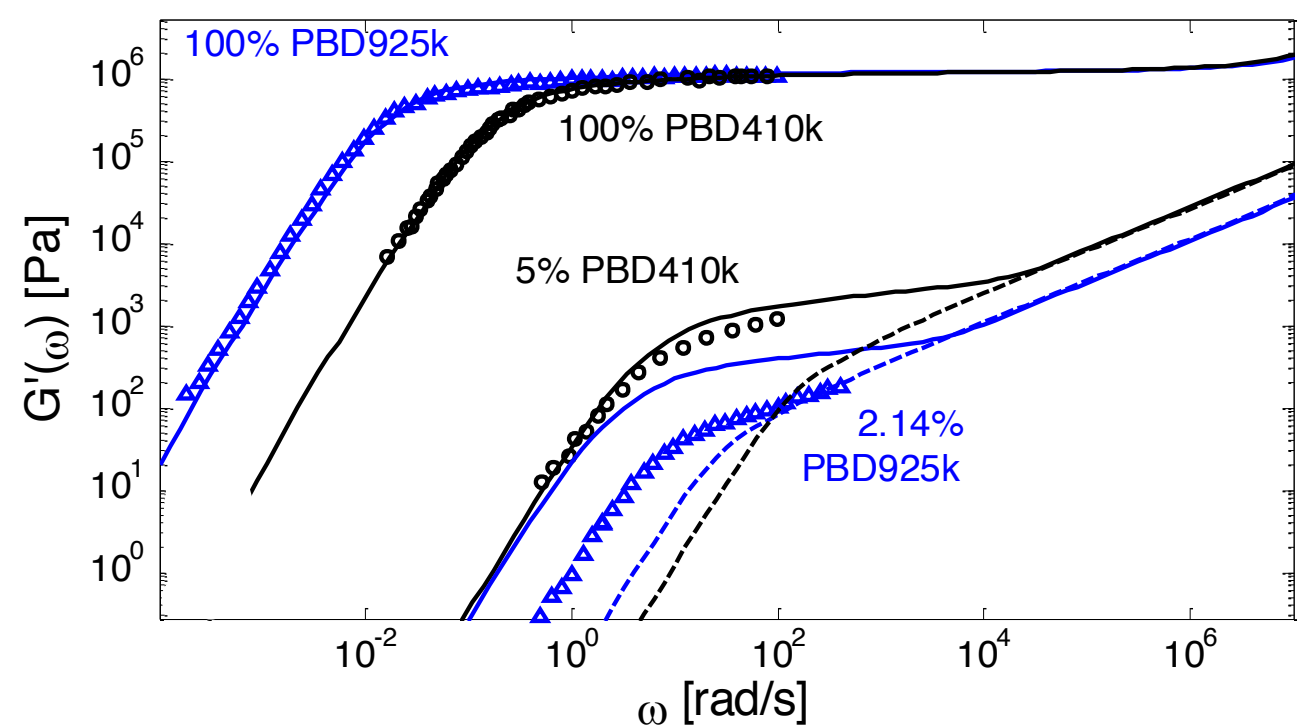

Figure 11: Experimental (o) and predicted (-) storage modulus for PBD samples with $M_{w}=410 \mathrm{~kg} / \mathrm{mol}$ 
and $925 \mathrm{~kg} / \mathrm{mol}$, in monodisperse state or diluted in oligomers (for PBD410) or in DOP (for PBD925). The dashed curves show the corresponding Rouse relaxation.

From this figure it is clear that the two solutions have a very different viscoelastic behavior: contrary to the viscoelastic response of the PBD925k diluted in DOP, the response of the PBD410k sample diluted in oligomers strongly differs from a Rouse-like response and is well described by our model with a real $\alpha$ exponent of 1 . Furthermore, the level of its plateau could not be captured by considering an $\alpha$ value of 1.3. Indeed, in such as case, the storage plateau would have been 2.5 times lower than the present plateau, which does not correspond to the experimental data.

One can also observe that the two solutions have similar terminal relaxation times, which is somehow surprising since the reptation time of the diluted PBD925k should be higher by a factor of 4.9 (based on $\alpha=1$ ) or at least by a factor 3.8 (based on $\alpha=1.3$ ) than the reptation time of the PBD410k/PBD3k blend. This is also seen in Figure 8.b.: at low $Z_{2}$, the terminal relaxation times of the solutions in small-molecule solvent are much shorter than the relaxation times of all polymer/oligomers blends, as if the long chains were less entangled.

Other examples of PS solutions in small-molecule solvent can be found in literature. In particular, in ref. [34], Bhattacharjee et al. studied the rheology of PS chains of mass $\mathrm{M}_{\mathrm{w}}=3900 \mathrm{~kg} / \mathrm{mol}$ dissolved in diethyl phthalate (DEP), dibutyl phthalate (DBP) or dioctyl phthalate (DOP). However, due to the use of different solvents with different qualities (from good for DEP and DBP, to theta for DOP), it is difficult to determine the origin of the observed discrepancy for this last set of solutions. Another, systematic set of data has been presented by Osaki et al. ${ }^{19}$, who studied very long PS chains (with $\mathrm{M}_{\mathrm{w}}=2890,4480$ and 8420 $\mathrm{kg} / \mathrm{mol}$ ) dissolved in a good solvent (chlorinated biphenyl). Similarly to the results found with the PBD solutions, these curves cannot be modelled based on the approach used for the polymer/oligomers blends. This is illustrated in Figure 12, which compares experimental and predicted curves for the PS solution containing $5 \mathrm{wt} \%$ of PS8420: since $Z_{2}$ is equal to 28 (with $\alpha=1$ ), the predicted dilution effect is very small and at intermediate frequency, the storage modulus is close to its rubbery plateau, which has been determined based on $\alpha=1$. In contrast, we observe that the experimental plateau corresponds rather to an $\alpha$ value close to 1.3 , which 
is the scaling found by Osaki et al., after having removed the high frequency Rouse contribution from the experimental data in order to determine $G_{N}^{0}$.

On the other hand, the shape of the moduli is similar to the ones found for the blend composed of $40 \%$ of PS 820 in OS8.8 (see the dashed curves compared to the star symbols), i.e. corresponds to long chains with 22 entanglements. For PS8420 diluted at $5 \mathrm{wt} \%$, a value of $Z_{2}$ equal to 22 corresponds to an $\alpha$ value close to 1.08 (since $8420 \mathrm{k} /\left(M_{e 0 \text {, melt }} / 0.05^{1.08}\right)=22$ ). Thus, for this sample, it seems that the scaling in term of plateau modulus and relaxation time is different.

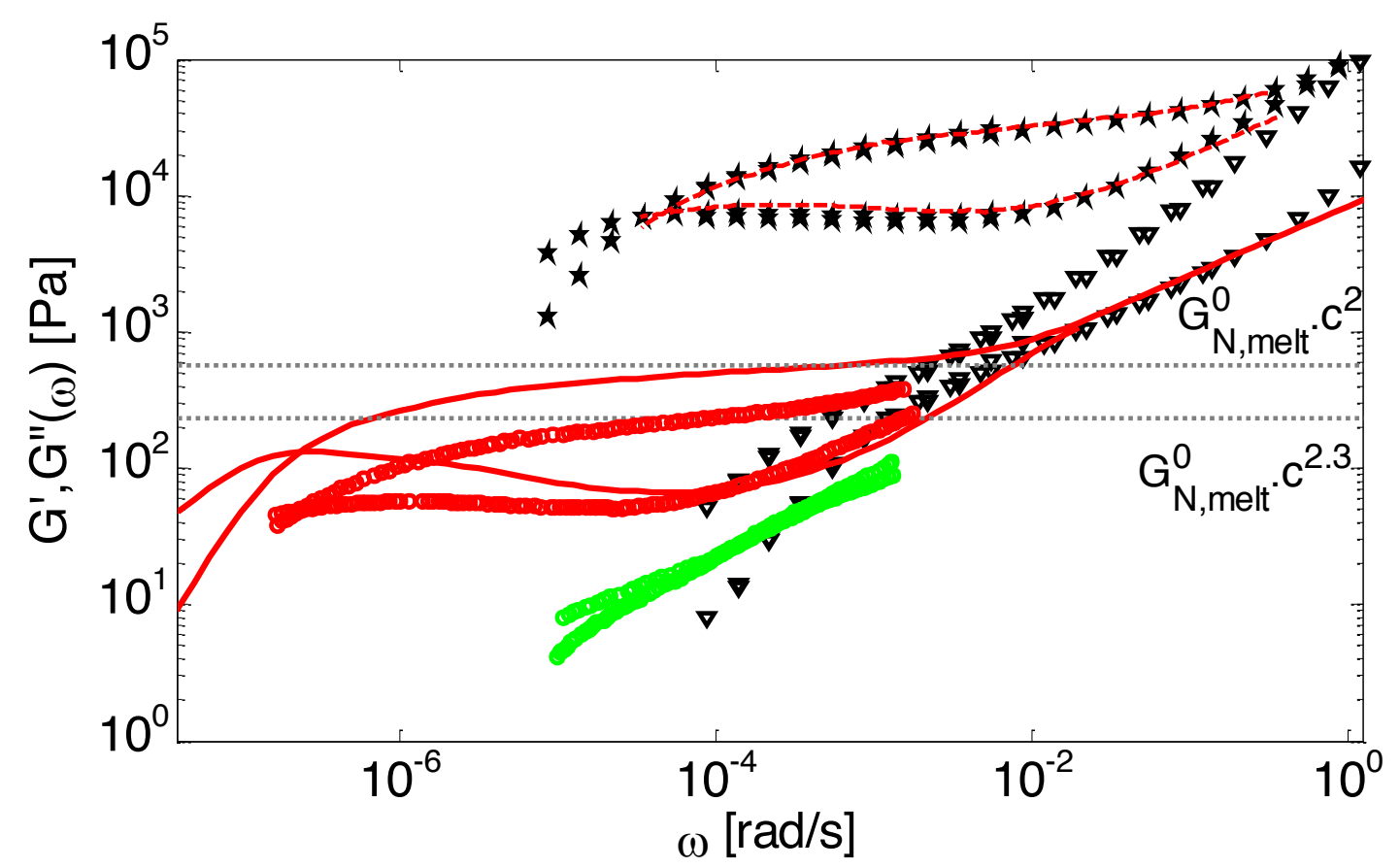

Figure 12: Comparison between the experimental storage and loss moduli of the blends PS820/OS8.8 containing $40 \mathrm{wt} \%\left(^{*}\right)$ or $5 \mathrm{wt} \%$ ( $\left.\nabla\right)$ of long chains, and the (horizontally shifted) moduli of sample PS8420 diluted at 5wt\% (red o) or 2wt\% (green o) in good solvent. The thick curves represent the predicted $G$ ' and $G$ " of sample PS8420 diluted at $5 \mathrm{wt} \%$, while the dashed curves represent the experimental data of this sample, which have been horizontally and vertically shifted in order to superimpose the data of the blend composed of $40 \mathrm{wt} \%$ PS820 in OS8.8.

The reason why the diluted PBD925k behaves differently compared to the polymer/oligomers blends is not clear and should be further investigated. While it could come from the fact the solvent used is a small-molecule solvent, which possibly affects the ability of the chains to 
create entanglements, it could also be due to a difference in the cooperativity between the long chains and the oligomers or the small-molecule solvent: the high-M chains in the smallmolecule solvent determine their entanglement mesh size just according to the solubility of the solvent, without any time needed to reach their equilibrium state. Thus, the high-M chains in those solutions obey the Colby-Rubinstein argument and their plateau modulus is proportional to $\mathrm{c}^{2.3}$ in semi-dilute solutions. In contrast, in the high-M/oligomers blends, there is a very strong cooperativity between the oligomers and high-M chains, at least up to the length scale of the oligomer chains, since the motion of the Rouse segments of the high-M chain occurs together with that motion of the oligomers. Consequently, this strong cooperativity leads to a slower equilibration, which is well described by the CR-CLF process. In order to validate this proposition, one should compare these data to the viscoelastic behavior of very long chains (similar to PS8420k) blended in few percent to short chains or oligomers and check if their effective $\alpha$ continue to be equal to 1 until $Z_{2}=12$ (as the theoretical values shown for PS4000 in Figure 9.a).

\section{Conclusions}

We have studied and modelled the viscoelastic properties of several polymer/oligomers blends, with various molar masses and concentrations of long chains, in order to discuss the dilution effect from the oligomers on the entanglement state of the long chains. In particular, we have shown that, at low concentration, the effective dynamic dilution exponent determined from the scaling of level of the storage modulus corresponding to $G_{\delta}$ or $G{ }^{\prime}{ }_{\max }$ with concentration is larger than the real or actual dilution exponent $\alpha$, which is fixed to 1 . This is due to the fast contribution of the CR-CLF process, which can take place without any delay coming from the oligomers. Consequently, in case that the number of (long-long) entanglements of the long chain falls below 12 (either by lowering the molecular weight or the solution concentration), $G_{\delta}$ is found to scale with $\mathrm{c}^{2.3}$ and cannot be used as a measure of the plateau modulus.

These results are in good agreement with ref. [11], where a larger value of the effective $\alpha$ in binary blends of linear chains was attributed to the partial re-orientation of the long-long entanglement segments, taking place through the tension re-equilibrium process along the chains. A similar picture is used here to analyze the viscoelastic properties of the 
polymer/oligomers blends, by considering that the relaxation time of a long-long entanglement segment is well described by its intrinsic Rouse time, with no extra delay due to the motion of the short chains.

While this approach could successfully explain the viscoelastic properties of a large number of polymer/oligomers blends, an important discrepancy was found for semi-dilute solutions of entangled polymers in theta or good solvents: their plateau moduli scaled with $\mathrm{c}^{2.3}$ rather than with $\mathrm{c}^{2}$. It has been shown that this behavior was not comparable to the viscoelastic behavior of the polymer/oligomers blends and that we could not describe the moduli of both types of samples with a single tube model. Possible origin of this difference has been proposed, based on the difference in cooperativity between the long chains and the oligomers or the smallmolecule solvent.

In perspective, one should study the viscoelastic properties of very long chains (i.e. still entangled when diluted at few percent) blended in short chains or oligomers, in order to validate our proposition. This question needs, indeed, to be solved if we want to compare the extensional behavior of these different blends and solutions.

\section{Acknowledgments}

The authors thank Prof. Hiroshi Watanabe for very helpful discussions and suggestions, Dr. Meik Ranft (BASF Ludwigshafen, Germany) for the determination of molecular weight distribution of the PS820 sample, and the People Programme (Marie Curie Actions) of the European Union's Seventh Framework Programme (REA grant agreement no. 607937). This work was funded by DSM Ahead (for T.S.), the Aage and Johanne Louis-Hansen foundation (for Q.H.) and the F.N.R.S.(for E.V.R as chercheur qualifié).

\section{References}

1. J.M. Dealy and R. G. Larson, Structure and rheology of molten polymers, Hanser Publishers, Munich, 2006.

2. H. Watanabe, Prog. Polym. Sci., 1999, 24, 1253; T. C. B. McLeish, Adv. Phys., 2002, 51, 1379. 
3. E. van Ruymbeke, C. Y. Liu and C. Bailly, Quantitative tube model predictions for the linear viscoelasticity of linear polymers, Rheol. Rev., 2007, 53-134.

4. A. E. Likhtman and T. C. B. McLeish, Macromolecules, 2002, 35, 6332.

5. R. N. Khaliullin and J. D. Schieber, Macromolecules, 2010, 43, 6202.

6. Y. Masubuchi, H. Watanabe, G. Ianniruberto, F. Greco and G. Marrucci, Macromolecules, 2008, 41, 8275.; Y. Masubuchi, J. I. Takimoto, K. Koyama, G. Ianniruberto, G. Marrucci and F. J. Greco, Chem. Phys., 2001, 115, 4387.; Y. Masubuchi, G. Ianniruberto, F. Greco and G. Marrucci, J. Chem. Phys., 2003, 119, 6925.

7. S. Shanbhag and R. G. Larson, Macromolecules, 2004, 37, 8160.

8. M. E. Shivokhin, E. van Ruymbeke, C. Bailly, D. Kouloumasis, N. Hadjichristidis and A. E. Likhtman, Macromolecules, 2014, 47, 2451; A. E. Likhtman, S. K. Sukumaran and J. Ramirez, Macromolecules, 2007, 40, 6748.

9. J. Park and R. G. Larson, Rheol. Acta, 2005, 44, 319.; S. J. Park and R. G. Larson, Macromolecules, 2004, 37, 597.; Park, S. J. and Larson R. G., Journal of Rheology, 2006, 50, 21.

10. S. Wang, S.Q. Wang, A. Halasa and W. L. Hsu, Macromolecules, 2003, 36, 5355.

11. E. van Ruymbeke, V. Shchetnikava, Y. Matsumiya, and H. Watanabe, Macromolecules, 2014, 47, 7653.

12. G. Marrucci, J. Polym. Sci., Polym. Phys. Ed., 1985, 23, 159. ; R. C. Ball and T. C. B. McLeish, Macromolecules, 1989, 22, 1911.

13. M. Doi and S. F. Edwards, The Theory of Polymer Dynamics,Oxford, 1986.

14. R. Colby, L. Fetters, W. G. Funk and W. W. Graessley, Macromolecules, 1991, 24, 3873.

15. H. Watanabe, S. Ishida, Y. Matsumiya and T. Inoue, Macromolecules, 2004, 37, 6619.; H. Watanabe, S. Ishida, Y. Matsumiya and T. Inoue, Macromolecules, 2004, 37, 1937.

16. T. Sawada, X. Qiao and H. Watanabe, Nihon Reorojo Gakkaishi (J. Soc. Rheol. Japan), 2007, 35, 11; Y. Matsumiya, K. Kumazawa, M. Nagao, O. Urakawa and H. Watanabe, Macromolecules, 2013, 46, 6067.

17. R. H. Colby, and M. Rubinstein, Macromolecules 1990, 23, 2753.; M. Rubinstein, and R.H. Colby, Polymer Physics; Oxford University Press: New York, 2003.

18. V.R. Raju, E.V. Menezes, G. Marin and W.W. Graessley, Macromolecules 1981, 14, 1668.

19. D. Auhl, P. Chambon, T. C. B. McLeish, and D. Read, Phys. Rev. Lett., 2009, 103, 136001.

20. C. Das, N. J. Inkson, D. J. Read, M. A. Kelmanson and T. C. B. McLeish, J. Rheol., 2006, 50, 207. 
21. D. J. Read, D. Auhl, C. Das, J. den Doelder, M. Kapnistos, I. Vittorias and T. C. B. McLeish, Science, 2011, 333, 1871.

22. Z. W. Wang, X. Chen and R. G. Larson, J. Rheol., 2010, 54, 223.

23. E. van Ruymbeke, Y. Masubuchi and H. Watanabe, Macromolecules, 2012, 45, 2085.

24. M. Kapnistos, D. Vlassopoulos, J. Roovers and L. G. Leal, Macromolecules, 2005, 38, 7852.

25. M. Ahmadi, C. Bailly, R. Keunings, N. Nekoomanesh, H. Arabi and E. van Ruymbeke, Macromolecules, 2011, 44, 647.

26. H. Watanabe, O. Urakawa and T. Kotaka, Macromolecules, 1994, 27, 3525.

27. D.J. Read, K. Jagannathan, S.K. Sukumaran and D. Auhl, Journal of Rheology, 2012, 56, 823.

28. J. L. Viovy, M. Rubinstein, and R. H. Colby, Macomolecules, 1991, 24, 3587.

29. M Doi, W. W. Graessley, E. Helfand and D. S. Pearson, Macromolecules, 1987, 20, 1900.

30. Q. Huang, L. Hengeller, N. J. Alvarez and O. Hassager, Macromolecules 2015, 48, 4158.

31. Q. Huang, O. Mednova, H. K. Rasmussen, N. J. Alvarez, A. L. Skov, K. Almdal and O. Hassager, Macromolecules 2013, 46, 5026.

32. Q. Huang, N. J. Alvarez, Y. Matsumiya, H. K. Rasmussen, H. Watanabe and O. Hassager, ACS Macro Letters 2013, 2, 741.

33. J. K. Nielsen, H. K. Rasmussen, M. Denberg, K. Almdal and O. Hassager, Macromolecules, 2006, 39, 8844.; A. Bach, K. Almdal, H. K. Rasmussen and O. Hassager, Macromolecules 2003, 36, 5174.

34. P.K. Bhattacharjee, J.P. Oberhauser, G.H. McKinley, L.G. Leal and T. Sridhar, Macromolecules $2002,35,10131$.

35. M. L. Williams, R. F. Landel, and J. D. Ferry, J. Am. Chern. Soc., 1955, 77, 3701.

36. C.Y. Liu, J. He, R. Keunings and C. Bailly, Macromolecules 2006, 39, 8867.

37. E. van Ruymbeke, J. Nielsen and O. Hassager, J. Rheol., 2010, 54, 1155.

38. E. van Ruymbeke, C. Bailly, R. Keunings and D. Vlassopoulos, Macromolecules, 2006, 39, 6248.

39. Shchetnikava, V., J. J. M. Slot, and E. van Ruymbeke, Macromolecules, 2014, 47, 3350.

40. M. J. Struglinski and W. W. Graessley, Macromolecules, 1985, 18, 2630. 
41. P. E. Rouse, The Journal of Chemical Physics, 1953, 21, 1272.

42. Milner, S. T., T. C. B. McLeish, R. N. Young, A. Hakiki, and J. M. Johnson, Macromolecules, $1998,31,9345$.

43. E. van Ruymbeke, M. Kapnistos, D. M. Knauss and D. Vlassopoulos, Macromolecules, 2007, 40, 1713.

44. T. Inoue, H. Okamoto, and K. Osaki, Macromolecules, 1991, 24, 5670; E. van Ruymbeke, E.B.

Muliawan, D. Vlassopoulos, H. Gao and K. Matyjaszewski, European Polymer Journal, 2011, 47, 746.

45. C. Liu, J. He, E. van Ruymbeke, R. Keunings and C. Bailly, Polymer, 2006, 47, 4461.

46. Y. Matsumiya, N. Rakkapao and H. Watanabe, Macromolecules, 2015, 48, 7889.

47. E. van Ruymbeke, R. Keunings and C. Bailly, J. Non-Newtonian Fluid Mech., 2005, 128, 7.

48. E. van Ruymbeke, M. Kapnistos, D. Vlassopoulos, C. Y. Liu and C. Bailly, Macromolecules, 2010, 43, 525.

49. S. Costanzo, Q. Huang, G. Ianniruberto, G. Marrucci, O. Hassager and D. Vlassopoulos, Macromolecules, 2016, 49, 3925.

50. H. Tao, C. Huang and T.P. Lodge, Macromolecules 1999, 32, 1212.

51. J. W. Park, J. Yoon, J. Cha and H. S. Lee, Journal of Rheology, 2015, 59, 1173. 